\title{
Lymphotoxins Promote the Progression of Human Lymphatic Malformation by Enhancing Lymphatic Endothelial Cell Proliferation
}

\author{
Jie-Gang Yang, ${ }^{*}$ Yan-Fang Sun, ${ }^{* \dagger}$ Ke-Fei He, ${ }^{* \dagger}$ Jian-Gang Ren, ${ }^{* \dagger}$ Zhuo-Jue Liu, ${ }^{*}$ Bing Liu, ${ }^{* \dagger}$ Wei Zhang, ${ }^{* \dagger}$ and Yi-Fang Zhao
}

From the State Key Laboratory Breeding Base of Basic Science of Stomatology and Key Laboratory of Oral Biomedicine Ministry of Education* and the Department of Oral and Maxillofacial Surgery, ${ }^{\dagger}$ School and Hospital of Stomatology, Wuhan University, Wuhan, China

Accepted for publication July 26, 2017.

Address correspondence to Yi-Fang Zhao, M.S., or Wei Zhang, Ph.D., The State Key Laboratory Breeding Base of Basic Science of Stomatology and Key Laboratory of Oral Biomedicine Ministry of Education, School and Hospital of Stomatology, Wuhan University, Wuhan 430079, China. Email: yifang@whu.edu.cn or wzhang88@whu.edu.cn.

\begin{abstract}
Formation of inflammation-related tertiary lymphoid organs promotes human lymphatic malformation (LM) development. However, the role of lymphotoxins (LTS) and LT-related inducible ligand, the crucial mediators for tertiary lymphoid organ formation, is undetermined in LMs. Herein, we show that LTs and LT-related inducible ligand promote LM development by enhancing lymphatic endothelial cell (LEC) proliferation via activating NF- $\kappa B$ pathways. The expression of LTs and their receptors was increased in LMs, especially the infected ones, when compared with normal skins. Nuclear translocation of p65, p52, and RelB in the LECS of LMs indicated the activation of classic and alternative NF- $\kappa B$ pathways. Pearson's correlation and cluster analysis suggested the close relationship between LEC proliferation and NF- $\kappa B$ activation. Moreover, in vitro data demonstrated LTs accelerated the proliferation of human dermal LECs (HdLECS) through activation of NF-KB. In addition, lipopolysaccharide (LPS) up-regulated LT receptor expression in HdLECs, leading to increased sensitivity to LTs. Suppression of LT receptors hampered LPS-enhanced HdLEC proliferation, indicating the crucial role of LT pathways in inflammatory lymphangiogenesis. Besides, evidence from the LM rat models demonstrated LT $\alpha$ and LPS enhanced LEC proliferation, therefore promoting LM development. Blocking LT pathways by neutralizing antibodies against $L T \alpha$ and lymphotoxin $\beta$ receptor may decelerate the growth of the disease. In summary, our present study demonstrated activation of LT signaling pathways in LECs contributed to the progression of LMs. (Am J Pathol 2017, 187: 2602-2615; http://dx.doi.org/10.1016/j.ajpath.2017.07.019)
\end{abstract}

Lymphatic malformations (LMs) are slow-flow vascular anomalies, arising from abnormal development of lymphatic channels, ${ }^{1}$ and characterized by dilated and enlarged lymphatic vessels. ${ }^{2}$ LMs are classified into three subtypes, according to the size of their lymphatic vessel lumen. The diameter of lumen in microcystic LMs is $<1 \mathrm{~cm}$, whereas that in macrocystic LMs is $>1 \mathrm{~cm}$. And the mixed LMs are composed of microcystic and macrocystic lesions. ${ }^{3}$ Most LMs occur in head and neck regions ${ }^{4}$; thus, they may cause cosmetic problems and even threaten patient lives when obstructing the airway. Current therapies for LMs include surgery, sclerotherapy, and laser treatment. ${ }^{5-7}$ These therapies are not effective all of the time, especially for the microcystic lesions. ${ }^{8}$ Therefore, mechanisms underlying the development of LM need to be explored for developing efficient therapies.
Clinical observation indicates that chronic inflammation, which is usually associated with injuries or upper respiratory tract infection, is a common symptom observed with the progression of LMs. ${ }^{9}$ Our previous study has demonstrated that LMs with infection showed enhanced lymphangiogenesis. ${ }^{10}$ Moreover, consistent with the study from other investigators, ${ }^{11}$ our research ${ }^{10}$ has revealed that tertiary lymphoid organs (TLOs), which were composed of aggregated lymphocytes in a highly organized manner and

\footnotetext{
Supported by the National Natural Science Foundation of China grant 81371159 (Y.-F.Z.), 81502708 (Y.-F.S.), and 81602780 (W.Z.), and the Young Elite Scientist Sponsorship Program by China Association for Science and Technology (CAST) 2015QNRC001 (Z.W.).

J.-G.Y. and Y.-F.S. contributed equally to this work.

Disclosures: None declared.
} 
resembled normal secondary lymphoid organs (eg, lymph nodes), were commonly seen in human LMs. In addition, the TLOs contribute to new lymphatic vessel formation. ${ }^{12}$ Lymphotoxins (LTs) are the vital cytokines that mediated the formation of TLOs during chronic inflammation. ${ }^{13,14}$ Homotrimer $\mathrm{LT}_{3}$ and membrane-bound heterotrimer LT $\alpha_{1} \beta_{2}$, composed by the monomers LT $\alpha$ and LT $\beta$, respectively, are the two main members of the lymphotoxin family, ${ }^{15}$ which belong to the tumor necrosis factor (TNF) superfamily. ${ }^{16}$ LIGHT (LT-related inducible ligand that competes for glycoprotein D binding to herpesvirus entry mediator on $\mathrm{T}$ cells), also known as TNFSF14 (TNF superfamily member 14), is homologous to LTs. ${ }^{15}$ Previous studies have reported that LTs and LIGHT promote the formation of high endothelial venules and lymphatic vessels in TLOs. ${ }^{17-19}$ Moreover, LT $\alpha$ contributes to increased lymphatic vessel formation within TLOs and, therefore, promotes the drainage of tissue fluids. ${ }^{20}$ In addition, activation of lymphotoxin $\beta$ receptor (LT $\beta R$ ) in endothelial cells has been revealed to facilitate the development of vasculatures and maintain their homeostasis, ${ }^{21}$ as well as mediate the lymphocyte transendothelial migration via upregulation of CXCL12. ${ }^{22}$ However, whether LTs and LIGHT are involved in the progression of LMs, especially in these lesions with infection, is still unknown.

Once LTs and LIGHT bind to their receptors on the membrane, the intracellular NF- $\kappa \mathrm{B}$ pathways are activated, mediating the LT-related biological processes. Generally, the NF- $\kappa \mathrm{B}$ signaling pathway occurs through either a classic (canonical) or an alternative (noncanonical) pathway. ${ }^{23}$ In the classic NF- $\kappa \mathrm{B}$ pathway, I $\kappa \mathrm{B}$ kinase is activated and then phosphorylates I $\mathrm{K} \mathrm{B} \alpha$ together with I $\mathrm{K} B$ kinase $\gamma$, which further induces the nuclear translocation of heterodimers p50/p65; in the alternative NF- $\kappa B$ pathway, NF$\kappa \mathrm{B}-$ inducing kinase and IкB kinase $\alpha$ are activated and then phosphorylate $\mathrm{p} 100$, which leads to nuclear translocation of p52/RelB. ${ }^{22,23}$ The classic NF- $\mathrm{BB}$ pathway, commonly stimulated by tumor necrosis family members, including $\mathrm{LT}_{3}$, is considered to be the most important transduction pathway in various pathological conditions. $^{23,24}$ Alternative NF- $\kappa \mathrm{B}$ signaling, which can be activated by a limited number of LT $\beta$ R ligands, is mainly involved in the activation of immunocytes, including B-cell survival and maturation, and dendritic cell activation. ${ }^{25}$ Despite the acknowledgment concerning the function of both classic and alternative NF- $\mathrm{BB}$ signaling pathways in lymphoid organogenesis, ${ }^{14,20}$ whether and which NF- $\mathrm{BB}$ pathway is activated in human LMs remain to be elucidated.

Herein, we detected the expression and distribution of LTs, LIGHT, and their receptors in human LM tissues. Moreover, the activation of crucial downstream factors of LT signaling pathways, including classic and alternative NF- $\kappa \mathrm{B}$ pathways in LMs, has been explored. In addition, cluster analysis was performed to analyze the correlation between the activation of LT pathways in the LM specimens and their infection status. The increased expression of LTs, LIGHT, and their receptors was also detected in human dermal lymphatic endothelial cells (HdLECs) after stimulation of lipopolysaccharide (LPS). Moreover, LTs and LIGHT activated the NF- $\mathrm{BB}$ pathways and enhanced the proliferation of HdLECs. Finally, the effects of LPS and LT $\alpha$ on the progression of LMs were evaluated in an LM rat model.

\section{Materials and Methods}

\section{Samples and Immunohistochemistry}

Six samples of normal human skin and 20 clinical samples of LMs were collected from the Hospital of Stomatology, Wuhan University (Wuhan, China). Seven of the LM samples were associated with infection. The infection status of the samples was confirmed by the pathologists (K.-F.H. and J.-G.R.) on the basis of the pathological characteristics. The six samples of normal skin were collected during the repair of cleft lip at the Hospital of Stomatology, Wuhan University. The relative information for tissue samples is listed in Supplemental Tables S1 and S2. All of the procedures and protocols were performed in accordance with the $\mathrm{NIH}$ guidelines regarding the use of human tissues and approved by the review board of the Ethics Committee of the Hospital of Stomatology, Wuhan University. Written informed consent for this study was obtained from all of the participants. The diagnosis of each LM case was performed by two independent pathologists (K.-F.H. and J.-G.R.), according to the histopathological features after hematoxylin and eosin staining. The immunohistochemistry was performed in accordance with our previous procedures. ${ }^{26}$ Briefly, the serial tissue sections of formalin-fixed, paraffin-embedded tissues were dewaxed in xylene and rehydrated in ethanol and doubledistilled water. The antigen was retrieved by high pressure. Then, the sections were incubated within 3\% hydrogen peroxide and $10 \%$ goat serum for 15 minutes, respectively. After that, the slides were incubated overnight at $4{ }^{\circ} \mathrm{C}$ with primary antibodies: LT $\alpha$ (mouse monoclonal; 1:200; BMS 105; eBioscience, San Diego, CA), LT $\beta$ (rabbit polyclonal; 1:200; orb 100093; Biorbyt, Cambridge, UK), LIGHT (rabbit polyclonal; 1:400; orb 1725; Biorbyt), TNF receptor (TNFR) 1 (rabbit polyclonal; 1:200; 21574-1-AP; Proteintech, Chicago, IL), TNFR2 (rabbit polyclonal; 1:200; 19272-1-AP; Proteintech), LT $\beta R$ (rabbit monoclonal; 1:400; 20331-1-AP; Proteintech), p65 (rabbit polyclonal; 1:400; 10745-1-AP; Proteintech), p52 (rabbit polyclonal; 1:400; 10409-1-AP; Proteintech), prospero homeobox 1 (PROX1; rabbit polyclonal; 1:100; 51043-1-AP; Proteintech), RelB (rabbit monoclonal; 1:200; number 4922; Cell Signaling Technology, Danvers, MA), Ki-67 (mouse monoclonal; 1:200; number 9449; Cell Signaling Technology), lymphatic vessel endothelial hyaluronan receptor 1 (rabbit polyclonal; 1:200; ab14917; Abcam, Cambridge, UK), and Fms-related tyrosine kinase 4 (rabbit polyclonal; 1:50; A5605; Abclonal, Cambridge, MA). After incubating with horseradish peroxidase-conjugated secondary antibody (goat anti-mouse and goat anti-rabbit; 
Kit-5001 and Kit-5004; Maixin, Fuzhou, China), the antibody binding was detected and visualized using a diaminobenzidine substrate kit (Dako, Glostrup, Denmark).

\section{Evaluation of Immunohistochemical Staining}

Five randomly selected images of each slide were taken using an Olympus microscope (BX51; Olympus, Tokyo, Japan) at a magnification of $\times 200$, and then evaluated by two investigators (J.-G.Y and W.Z.) blindly, without knowledge of the clinicopathological parameters. The staining of LTs, LIGHT, and their receptors were mainly localized within the cytoplasm. The staining of RelB, p52, and p65 mainly localized within the cytoplasm and/or nucleus, and the nuclear localization was considered as activation. Histoscores ( 0 to 7$)$ were calculated as the summation of staining intensity ( 0 to 3 ) and the ratio of positive stained LECs/PROX1-positive total LECs (0 to 4$)$. The staining intensity was ranked from 0 to 3 ( 0 , negative; 1 , mild staining; 2 , moderate staining; and 3 , intense staining.) The ratio of positive-stained cells was scored from 0 to $4(0,<10 \% ; 1, \geq 10 \%$ to $<25 \% ; 2, \geq 25 \%$ to $<50 \% ; 3$, $\geq 50 \%$ to $<75 \%$; and $4, \geq 75 \%$ ). ${ }^{27}$ The staining intensity and the ratio of positive staining were determined blindly by the two investigators (J.-G.Y and W.Z.).

\section{ELISA Data}

Total tissue lysates from $100 \mathrm{mg}$ clinical LM samples and normal skin tissues were collected by harvesting the supernatant of homogenized tissue lysates (the tissues were homogenized with $600 \mu \mathrm{L}$ ice-cold phosphate-buffered saline (PBS) buffer, followed by centrifugation at $15,000 \times g$ for $15 \mathrm{mi}-$ nutes). The concentration of LT $\alpha$, LT $\beta$, and LIGHT in tissue lysates was measured by enzyme-linked immunosorbent assay (ELISA) with human LT $\alpha$-specific (70-EK11392; MultiSciences Biotech, Hangzhou, China), LT $\beta$-specific (CSBE09980h; Cusabio, College Park, MD), and LIGHT-specific (70-EK12171; MultiSciences Biotech) ELISA kits. The concentration of proteins was standardized by total tissue protein.

\section{Multiple-Labeling Immunofluorescence}

Multiple labeling, including double- and triple-labeling immunofluorescence, was performed to evidence the coexpression of proteins. In brief, the tissue sections of paraffinembedded specimens were dewaxed in xylene and rehydrated in a graded series of ethanol and double-distilled water. Antigen retrieval was performed by high pressure. Blocking solution of $10 \%$ nonimmune donkey serum (Sigma-Aldrich, Seelze, Germany) was used for 1 hour at room temperature. The sections were subsequently incubated at $4^{\circ} \mathrm{C}$ overnight with primary antibodies: TNFR1 (rabbit polyclonal; 1:100; 21574-1-AP; Proteintech), TNFR2 (rabbit polyclonal; 1:100; 19272-1-AP; Proteintech), LT $\beta R$ (rabbit monoclonal; 1:200; 20331-1-AP; Proteintech), p65 (rabbit polyclonal; 1:200;
10745-1-AP; Proteintech), p52 (rabbit polyclonal; 1:200; 10409-1-AP; Proteintech), PROX1 (rabbit polyclonal; 1:50; 51043-1-AP; Proteintech), RelB (rabbit monoclonal; 1:100; number 4922; Cell Signaling Technology), Ki-67 (mouse monoclonal; 1:100; number 9449; Cell Signaling Technology), CD3 (mouse monoclonal; 1:100; kit-0003; Maixin), and CD20 (mouse monoclonal; 1:100; kit-0001; Maixin). This was followed by incubation of secondary antibodies: DyLight 488 (goat anti-mouse and goat anti-rabbit; ab96879 and ab96899; Abcam) or DyLight 594 (goat anti-mouse and goat anti-rabbit; ab96881 and ab96885; Abcam). Nuclei were counterstained with DAPI (ZLI-9557; ZSGB-Bio, Beijing, China), followed by observation under a fluorescence microscope (Leica DM4000B; Leica, Wetzlar, Germany).

\section{Hierarchical Clustering and Data Visualization}

According to our previous study, ${ }^{28}$ the staining scores of immunohistochemistry were turned into scaled values centered on 0. Hierarchical analysis on the basis of Pearson's correlation coefficients was performed by Cluster 3.0 (Human Genome Center, University of Tokyo, Tokyo, Japan). The clustered results were presented using Java TreeView 1.0.5 (Free Software Foundation, Inc., Boston, MA). The biomarkers and specimens were arranged on the horizontal and vertical axes, respectively. Two closely related biomarkers are located with each other.

\section{Cell Culture}

HdLECs were purchased from ScienCell (Carlsbad, CA). HdLECs were cultured at $37^{\circ} \mathrm{C}$ with $5 \% \mathrm{CO}_{2}$ in endothelial cell medium (ScienCell) supplemented with $20 \%$ fetal bovine serum, $100 \mathrm{U} / \mathrm{mL}$ penicillin, $100 \mathrm{ng} / \mathrm{mL}$ streptomycin, and $100 \mu \mathrm{g} / \mathrm{mL}$ endothelial cell growth supplement (ScienCell). HdLECs were treated with $1 \mu \mathrm{g} / \mathrm{mL}$ LPS (Sigma-Aldrich, St. Louis, MO), recombinant human $\mathrm{LT} \alpha_{3}$ (R\&D Systems, Minneapolis, MN) and $100 \mathrm{ng} / \mathrm{mL} \mathrm{LT} \alpha_{1} \beta_{2}$ (R\&D Systems), or $100 \mathrm{ng} / \mathrm{mL}$ recombinant human LIGHT (Peprotech, Rocky Hill, NJ) in endothelial cell medium containing $2 \%$ fetal bovine serum and further cultured for 24 hours.

\section{RNA Interference}

Cells were cultured in 6-well plates at a density of $50 \%$ confluence 18 hours before transfection. Transfection was performed with Transfection Reagent (Biotool, Houston, TX), according to the instructions. Cell lysate was harvested 24 hours after transfection of siRNA. TNFR1, TNFR2, and LT $\beta$ R siRNAs were purchased from Ribobio Co, Ltd (Guangzhou, China).

\section{Real-Time Quantitative PCR}

According to our previous study, ${ }^{29}$ the isolation of total RNA, the synthesis of cDNA, and real-time quantitative 
PCR were conducted. Glyceraldehyde-3-phosphate dehydrogenase was chosen as the internal control in the following experiments. The primer nucleotide sequences for real-time quantitative PCR are listed in Table 1.

\section{Western Blot Analysis}

The Western blot analysis was performed according to our previous description. ${ }^{26}$ In brief, the proteins in corresponding cells were extracted, the nuclear fractions were prepared with the instructions of the Nuclear Extraction Kit (Sangon Biotech, Shanghai, China), and the protein concentration was estimated using bicinchoninic acid assay (Pierce, Rockford, IL). Subsequently, an aliquot of $20 \mu \mathrm{g}$ protein of each sample was separated on 10\% SDSpolyacrylamide gels and electroblotted on polyvinylidene fluoride membranes (Roche Applied Science, Penzberg, Germany). Then, the blots were blocked overnight with 5\% skimmed milk and further probed with primary antibodies: TNFR1 (rabbit polyclonal; 1:1000; 21574-1-AP; Proteintech), TNFR2 (rabbit polyclonal; 1:1000; 19272-1-AP; Proteintech), LT $\beta R$ (rabbit monoclonal; 1:1000; 20331-1AP; Proteintech), phosphorylated p65 (rabbit monoclonal; 1:1000; number 3033; Cell Signaling Technology), RelB (rabbit monoclonal; 1:1000; number 4922; Cell Signaling Technology), glyceraldehyde-3-phosphate dehydrogenase (mouse monoclonal; 1:1000; number 97166; Cell Signaling Technology), histone H3 (mouse monoclonal; 1:1000; number 3638; Cell Signaling Technology), and $\alpha$-tubulin (mouse monoclonal; 1:1000; number 3873; Cell Signaling Technology). Then, membranes were incubated with horseradish peroxidase-conjugated secondary antibodies at room temperature for 1 hour. After that, the immunoblots were detected using a chemiluminescence kit (Pierce).

\section{5-Ethyl-2'-Deoxyuridine Incorporation Assays}

Proliferation of HdLECs was determined by using the CellLight EdU Apollo 488 In Vitro Imaging Kit (Ribobio), according to the manufacturer's instructions.

\section{Establishment of Lymphatic Malformation Model in Rats}

According to our previous study, ${ }^{30}$ female Wistar rats $(250 \pm 30 \mathrm{~g})$ were purchased from the Center for Animal
Experiment of Wuhan University in pressurized ventilated cages under the institutional regulations. Animal handling and procedures were approved and overseen by the Ethics Committee for Animal Research, Wuhan University. The methods were performed in accordance with the approved guidelines and regulations. Freund's incomplete adjuvant (Santa Cruz Biotechnology, Dallas, TX) was emulsified with PBS, PBS containing $100 \mu \mathrm{g} / \mathrm{mL}$ LPS, or PBS containing $120 \mathrm{ng} / \mathrm{mL}$ recombinant $\mathrm{LT} \alpha_{3}$ for injections. Two injections of adjuvant ( 2 weeks apart) were conducted into the subcutis of rat neck. Injection of PBS only was used as negative control. LT $\alpha$ antibody (100 $\mu \mathrm{g} / \mathrm{mL} ;$ MAB621; R\&D Systems) and LT $\beta R$ antibody (100 $\mu \mathrm{g} / \mathrm{mL}$; AF629; R\&D Systems) were used to block the LT signaling pathways. Mouse IgG Isotype (number 5415; Cell Signaling Technology) was used as control. Lymphatic malformations in rats were examined for every week. After 2 months, the animals were euthanized and the cystic lesions were harvested for further detection.

\section{Statistical Analysis}

One-way analysis of variance, Student-Newman-Keuls test, and Spearman rank correlation test were performed in GraphPad Prism version 6.0 (GraphPad Software Inc., San Diego, CA) for statistical analysis. $P<0.05$ was considered statistically significant.

\section{Results}

Prominent Expression of LTs, LIGHT, and Their Receptors in Human LMs

ELISA was performed to compare the expression of LT $\alpha$, LT $\beta$, and LIGHT in three samples of LMs with infection, three uninfected LMs, and three normal skins. The concentrations of LT $\alpha, \mathrm{LT} \beta$, and LIGHT were significantly higher in LM tissue lysates than in normal skins (Figure 1A). Besides, our results revealed higher concentration of both LT $\alpha$ and LT $\beta$ in LMs, compared with that of LIGHT; however, all of them in normal skins were at a considerably low level. Consistent with results from ELISA, the mRNA expression levels of LT $\alpha$ and LT $\beta$ were much higher in LM tissues than those in normal skin tissues (Figure 1B). Results above indicated the potential role of LTs and LIGHT in LMs. Notably, the expression of LT $\alpha$, LT $\beta$, and LIGHT was

Table 1 Primer Sequences Used for Real-Time Quantitative PCR

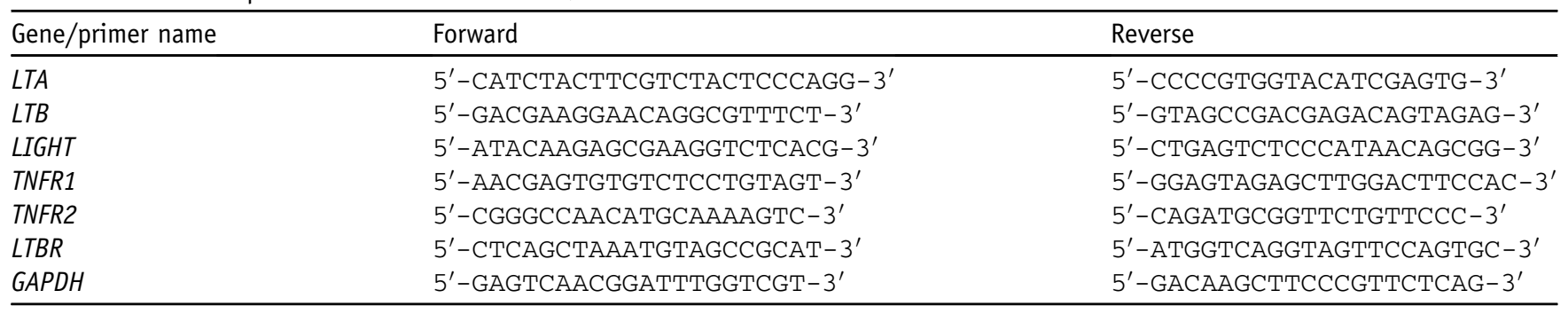


A

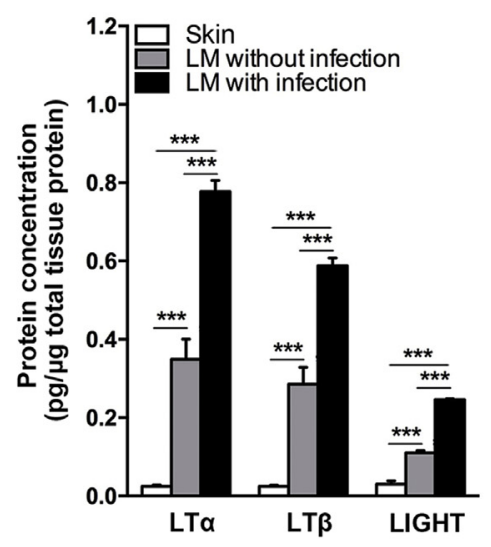

B

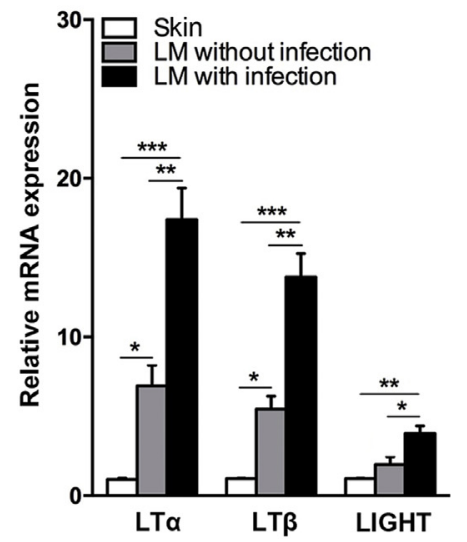

C

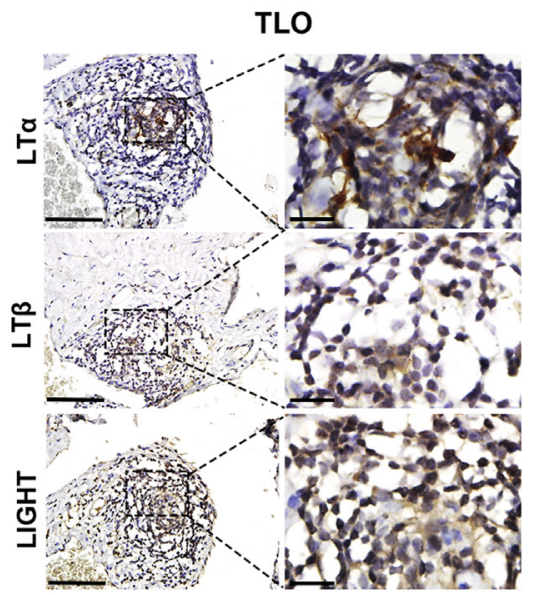

D
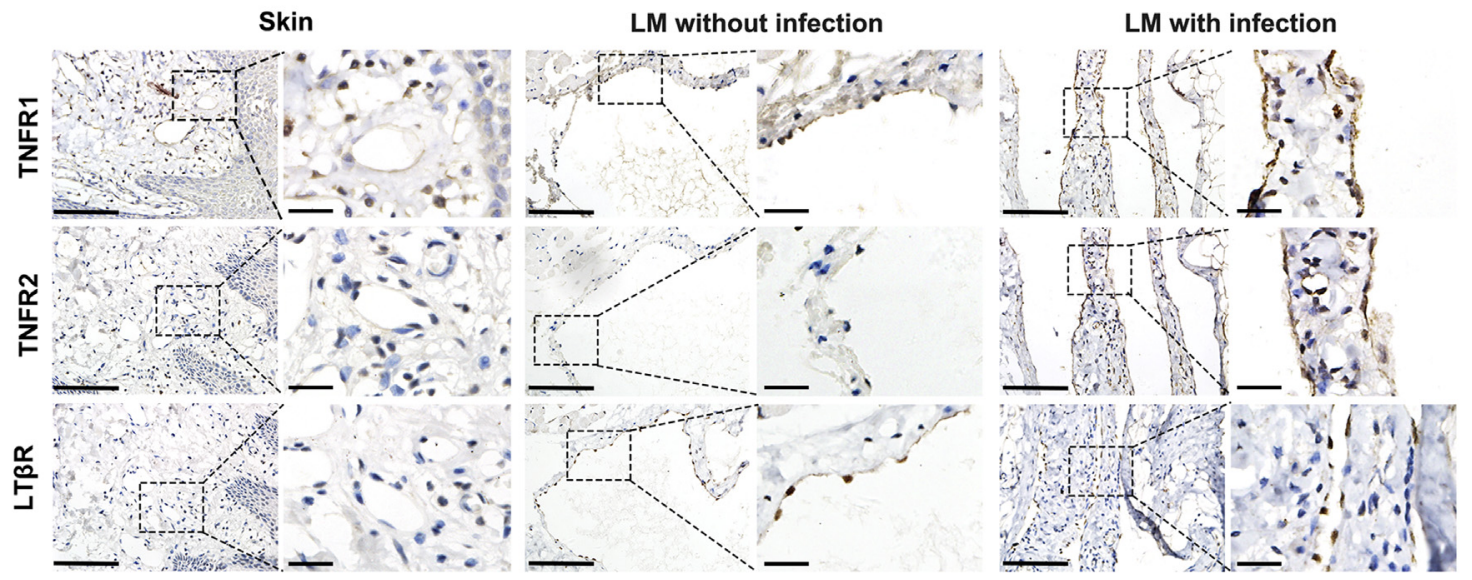

E

$\mathbf{F}$
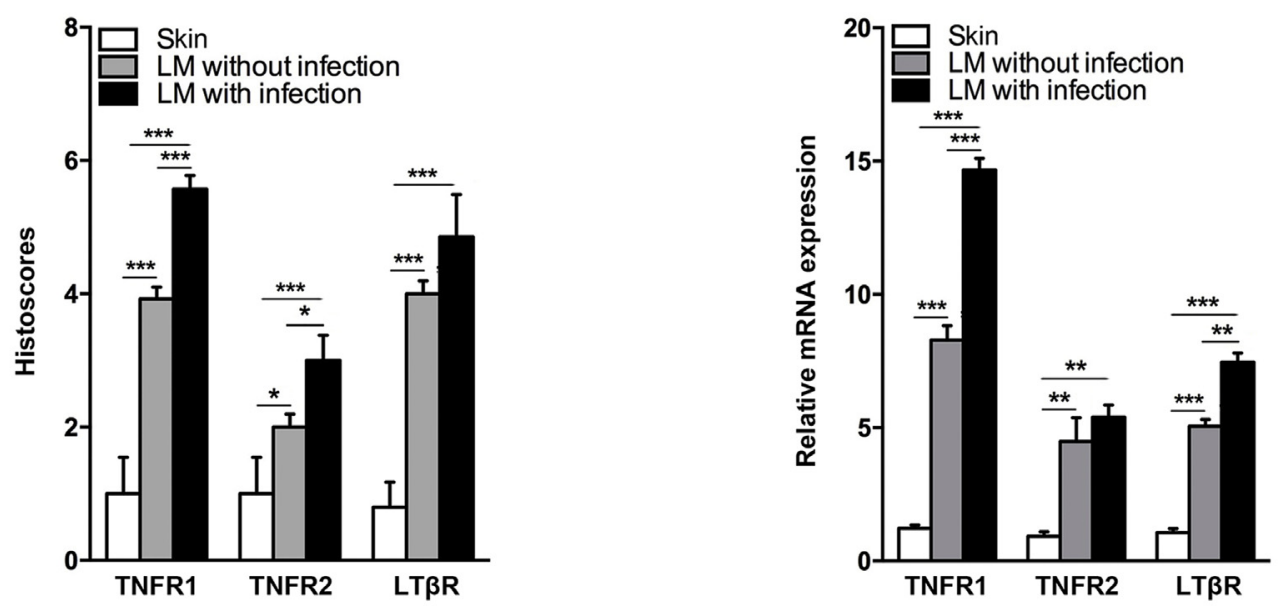

Figure 1 Expression of lymphotoxins (LTs) and their receptors in human lymphatic malformations (LMs). A: The protein expression levels of LT $\alpha$, LT $\beta$, and LT-related inducible ligand (LIGHT) in LMs were determined by enzyme-linked immunosorbent assay. Normal skins were used as control. B: mRNA expression of LT $\alpha$, LT $\beta$, and LIGHT in LM samples was detected by real-time quantitative PCR. C: Immunohistochemical staining of LT $\alpha$, LT $\beta$, and LIGHT in tertiary lymphoid organs (TLOs) in infected LMs. D: Increased expression of tumor necrosis factor receptor (TNFR) 1, TNFR2, and LT $\beta R$ in LMs. E: Histoscore of TNFR1, TNFR2, and LT $\beta R$ expression in LMs with or without infection and in skin samples. F: mRNA expression of TNFR1, TNFR2, and LT $\beta R$ was determined in LM samples by realtime quantitative PCR. Data are expressed as means $\pm \operatorname{SEM}(\mathbf{A}, \mathbf{B}, \mathbf{E}$, and $\mathbf{F}) . n=3(\mathbf{A}, \mathbf{B}$, and $\mathbf{F}) ; n=7$ (E, LMs with infection); $n=13(\mathbf{E}, \mathrm{LMs}$ without infection); $n=6$ (E, skin samples). ${ }^{*} P<0.05,{ }^{*} P<0.01$, and ${ }^{*} * P<0.001$. Scale bars: $50 \mu \mathrm{m}$ (C); $10 \mu \mathrm{m}$ (C, insets); $100 \mu \mathrm{m}$ (D); $20 \mu \mathrm{m}$ (D, insets). 
significantly higher in LMs with infection, compared with uninfected ones. Our previous study reported the potential role of TLO formation in LMs. ${ }^{10}$ Considering that LTs and LIGHT played essential roles in formation of TLOs, the expression of LTs and LIGHT was analyzed in TLOs of the infected LMs. Using antibodies against CD20, CD3, and peripheral node addressin, TLOs were identified with segregation of $\mathrm{B}$ and $\mathrm{T}$ cells and high endothelial venules (Supplemental Figure S1). Strong staining of LTs and LIGHT was detected in TLOs, indicating that TLOs might be the source of increased LTs and LIGHT in LMs (Figure 1C).

The channels positively stained with PROX1 in the human LM specimens were referred to as lymphatic vessels, because PROX1 is the most important transcription factor during the differentiation of LECs (Supplemental Figure S2). ${ }^{31}$ Meanwhile, lymphatic vessel endothelial hyaluronan receptor 1 and Fms-related tyrosine kinase 4 [alias vascular endothelial growth factor (VEGF) receptor 3] were taken as additional LEC markers. ${ }^{32-34}$ As LTs and LIGHT function through ligation to LT receptors, including TNFR1, TNFR2, and LT $\beta$, the expression levels of LT receptors were further evaluated in LM samples (Supplemental Table S1) and normal skins (Supplemental Table S2). The lymphatic vessels in human LM tissues exhibited an irregularly dilated shape compared with those in normal skin tissues (Figure 1D). The intensive staining of TNFR1 was observed in LM specimens (20/20), whereas only weak staining of TNFR1 was found in normal skin tissues $(6 / 6 ; P<0.001)$. Meanwhile, the slight staining of TNFR2 was noticed in human LMs, but completely undetectable in normal skin tissues. LT $\beta R$, the specific receptor for the LT $\alpha \beta$ heterotrimers and LIGHT, was highly expressed in LM tissues (20/20) compared with normal skin tissues. The histoscores and the mRNA expression of these receptors showed the increase of the receptors in LMs, especially the infected ones (Figure 1, D-F). Taken together, high expression of LTs and LIGHT as well as their receptors in human LM tissues suggested that LTs and LIGHT were more prone to activate the LECs in LMs rather than those in normal skins. Notably, the expression levels of these three receptors were much higher in the infected LMs rather than the uninfected lesions, indicating the close relationship between LT signaling pathways and the inflammation status in LMs.

\section{Activation of NF- $\kappa$ B Pathway Might Promote LEC Proliferation in Human LMs}

Previous studies reported that LTs and LIGHT activated $\mathrm{NF}-\kappa \mathrm{B}$ signaling pathways in various inflammation models. ${ }^{22,35,36}$ Thus, we explored whether NF- $\kappa B$ pathways were activated in human LMs. To this end, the expression of p65, p52, and RelB, the key transcription factors of classic and alternative NF- $\kappa B$ pathways, ${ }^{22,23}$ were investigated in human LM and skin tissues. The expression of p65, p52, and RelB was higher in nuclei of LECs in human LMs, compared with normal skin tissues (Figure 2A). Immunofluorescence revealed the colocalization of $\mathrm{p} 52$ and RelB in nuclei of LECs (Figure 2B), indicating nuclear translocation of p52/RelB. The nuclear localization of p52/RelB and p65 confirmed the activation of both alternative and classic NF$\kappa \mathrm{B}$ pathways. Besides, according to immunohistochemical analysis, LECs lining the vessels of infected LMs had more positively stained nuclei with p65 and p52/RelB compared with those of uninfected LMs (Figure 2, A and C), suggesting the higher activation of both NF- $\kappa \mathrm{B}$ pathways in LECs in the infected LMs.

Previous studies proved the close relationship between lymphangiogenesis and NF- $\kappa \mathrm{B}$ pathways in various conditions. ${ }^{32,37}$ Thus, the correlation between the activation of NF$\kappa \mathrm{B}$ pathways and the proliferation of LECs was next explored. Our results revealed the increased expression of Ki67 in LECs in LMs, especially in the infected ones, compared with that in normal skins. Moreover, the Spearman rank correlation tests demonstrated that the expression of Ki-67 positively correlated with the nuclei expression of p65 $(P<0.001)$, p52 $(P<0.01)$, and RelB $(P<0.001)$, suggesting NF- $\kappa \mathrm{B}$ pathway activation might contribute to the proliferation of the LECs in LMs (Figure 2D). Furthermore, immunofluorescence images showed the colocalization of Ki67 and p52, as well as Ki-67 and p65, in nuclei of LECs in LMs (Supplemental Figure S3, A and B), further indicating the crucial role of NF- $\kappa \mathrm{B}$ pathways in LEC proliferation in LMs. Notably, an increased proportion of LECs with colocalization of NF- $\kappa B$ transcriptional factors (p65 or p52) and Ki-67 was observed in LMs with infection, compared with those without infection (Supplemental Figure S3, C and D). Almost no nuclear translocation of p65 or p52 was found in LECs in normal skin tissues. In summary, our results above suggested that the activation of NF- $\mathrm{B}$ pathways in human LMs might contribute to the progression of the disease by promoting LEC proliferation.

\section{Activation of NF- $\kappa$ B Pathway and Its Clinical Significance in Human LMs}

Cluster analysis was performed to delineate the relationship among the expression of LT $\alpha$, LT $\beta$, LIGHT, p65, p52, RelB, and $\mathrm{Ki}-67$ in human LMs and skin tissues. In addition, the clinical features of LM samples were listed beside the heat map. The samples were clustered into different groups, according to the expression pattern of the proteins (Figure 3). Most infected LMs were contained in one cluster, in which the expression levels of LTs, LIGHT, NF- $\kappa$ B transcriptional factors, and Ki-67 were much higher than those of most uninfected lesions. Conversely, normal skin tissues were distributed in another cluster, with low expression levels of LT $\alpha$, LT $\beta$, LIGHT, p65, p52, RelB, and Ki-67. Moreover, LT $\alpha$ clustered much closer to Ki-67 compared with the other LTs, suggesting LT $\alpha$ might play a more important role in LEC proliferation. Overall, the above results indicated that the activation of the classic NF- $\mathrm{B}$ pathway might be 
A

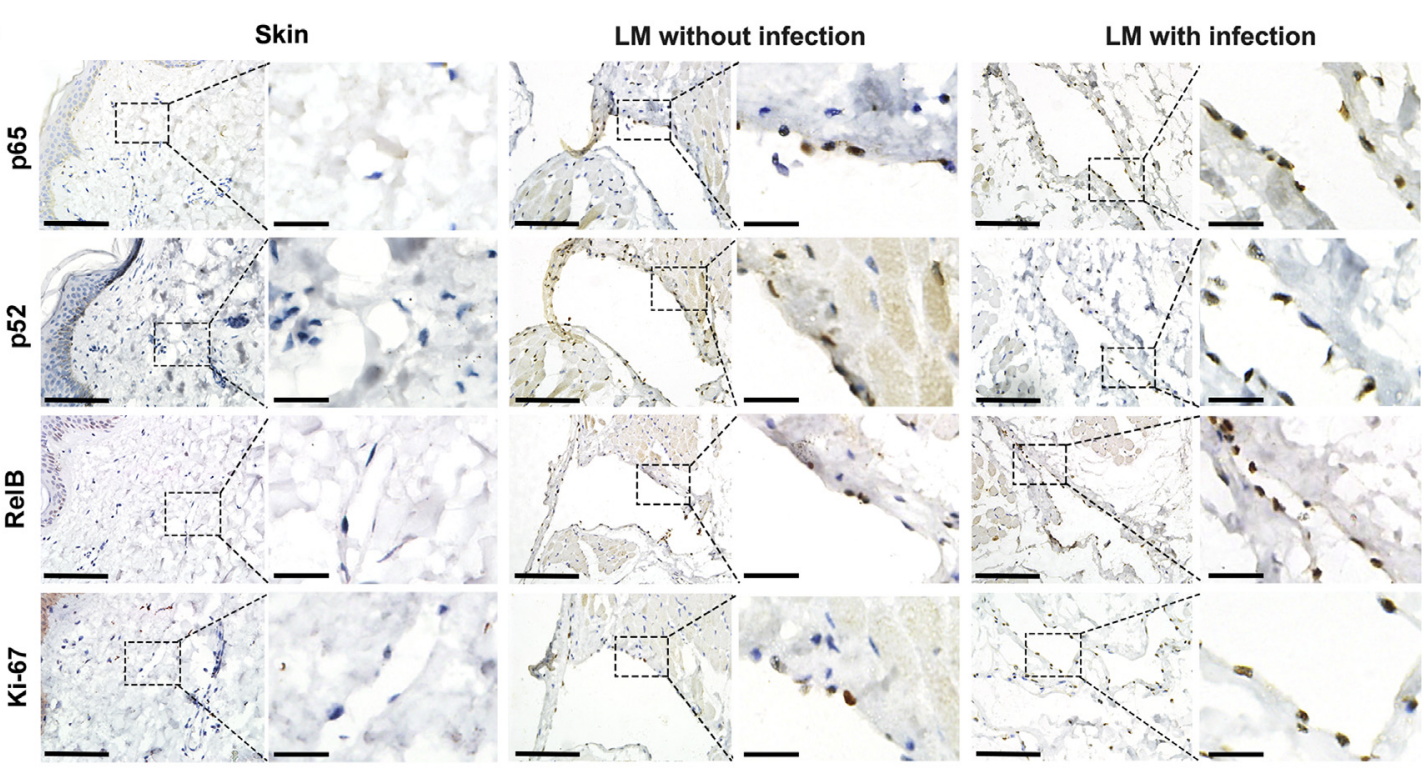

B
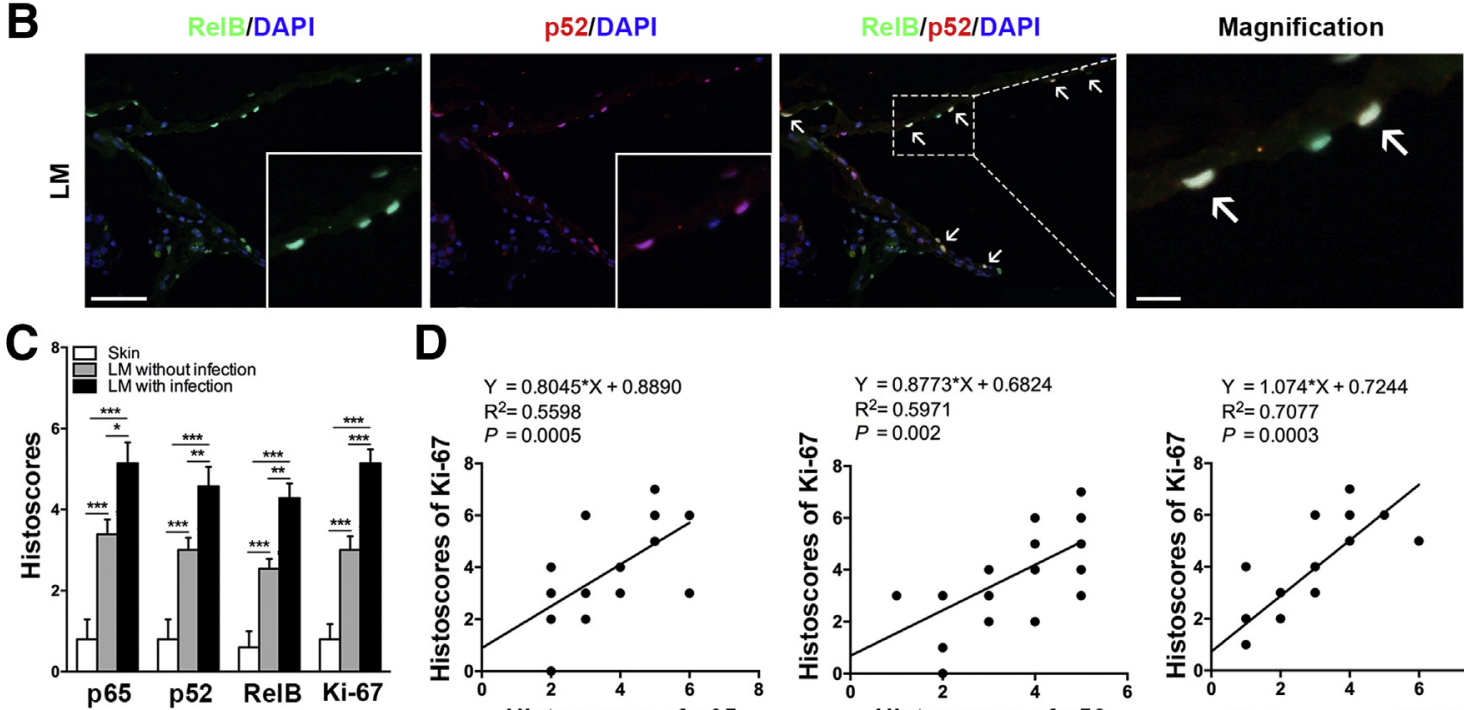

D $Y=0.8045^{*} X+0.8890$
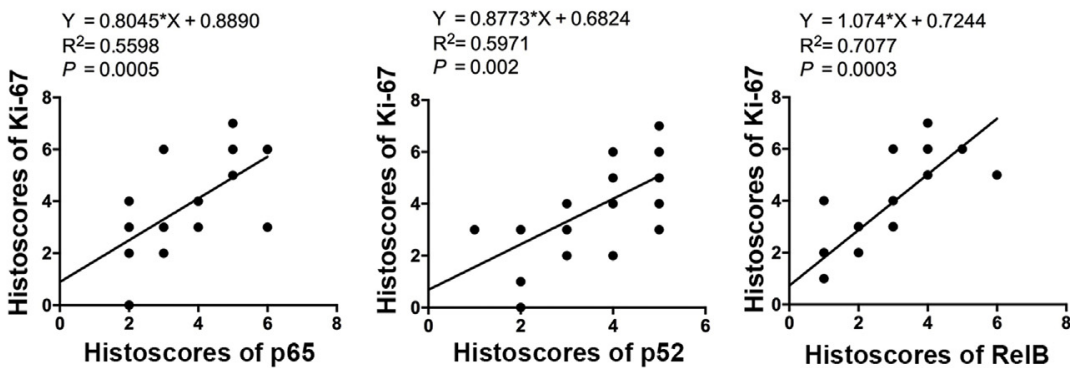

Figure 2 Correlation analysis of transcriptional factors p52, RelB, p65, and proliferation-related biomarker Ki-67. A: The expression of p65, p52, RelB, and Ki67 in human lymphatic malformations (LMs) was detected in serial sections. Normal skins were used as control. B: Double-labeling immunofluorescence staining for RelB and p52. Insets: Magnifications of local details. The arrows indicate RelB ${ }^{+} / \mathrm{p} 52^{+}$nucleus in lymphatic endothelial cells. C: Histoscores of p65, p52, RelB, and Ki-67 expression in infected and uninfected LMs and normal skin tissues. D: Spearman rank correlation analyses were performed to determine the relationship between Ki-67 and p65 and between p52 and RelB. Data are expressed as means \pm SEM (C). $n=7$ (C, infected LMs); $n=13$ (C, uninfected LMs); $n=6$ (C, normal skin tissues). ${ }^{*} P<0.05,{ }^{* *} P<0.01$, and ${ }^{* *} P<0.001$. Scale bars: $100 \mu \mathrm{m}$ (A); $20 \mu \mathrm{m}$ (A, insets); $50 \mu \mathrm{m}$ (B); $10 \mu \mathrm{m}$ (B, insets).

associated with LEC proliferation, whereas infection might lead to greater activation of the NF- $\mathrm{BB}$ pathway in LM lesions.

\section{LTs and LIGHT Promote HdLEC Proliferation by} Activating the Classic and Alternative NF- $\kappa$ B Pathways

We next explored whether LTs and LIGHT activated NF- $\kappa$ B pathways in LECs in vitro. Because only tripolymer can bind to their receptors and activate signaling pathways, recombinant $\mathrm{LT} \alpha_{3}$ and $\mathrm{LT} \alpha_{1} \beta_{2}$ were thus used. The HdLECs treated with LPS were used as positive control because previous studies demonstrated LPS can activate both classic and alternative NF-кB pathways. ${ }^{38,39}$ Nuclear translocation of p65 was observed in the recombinant $\mathrm{LT} \alpha_{3^{-}}$ treated HdLECs, indicating the activation of the classic NF$\kappa \mathrm{B}$ pathway (Figure 4A). Colocalization of p52 and RelB in nuclei was found in HdLECs that were treated with recombinant LT $\alpha_{1} \beta_{2}$ and LIGHT for 24 hours, suggesting the activation of the alternative NF- $\kappa \mathrm{B}$ signaling pathway.

Our results above revealed the increased expression of LT receptors in LECs in human LM tissues, especially under inflammation status. Thus, LPS, which was released from Gram-negative microbes, was used to explore whether 


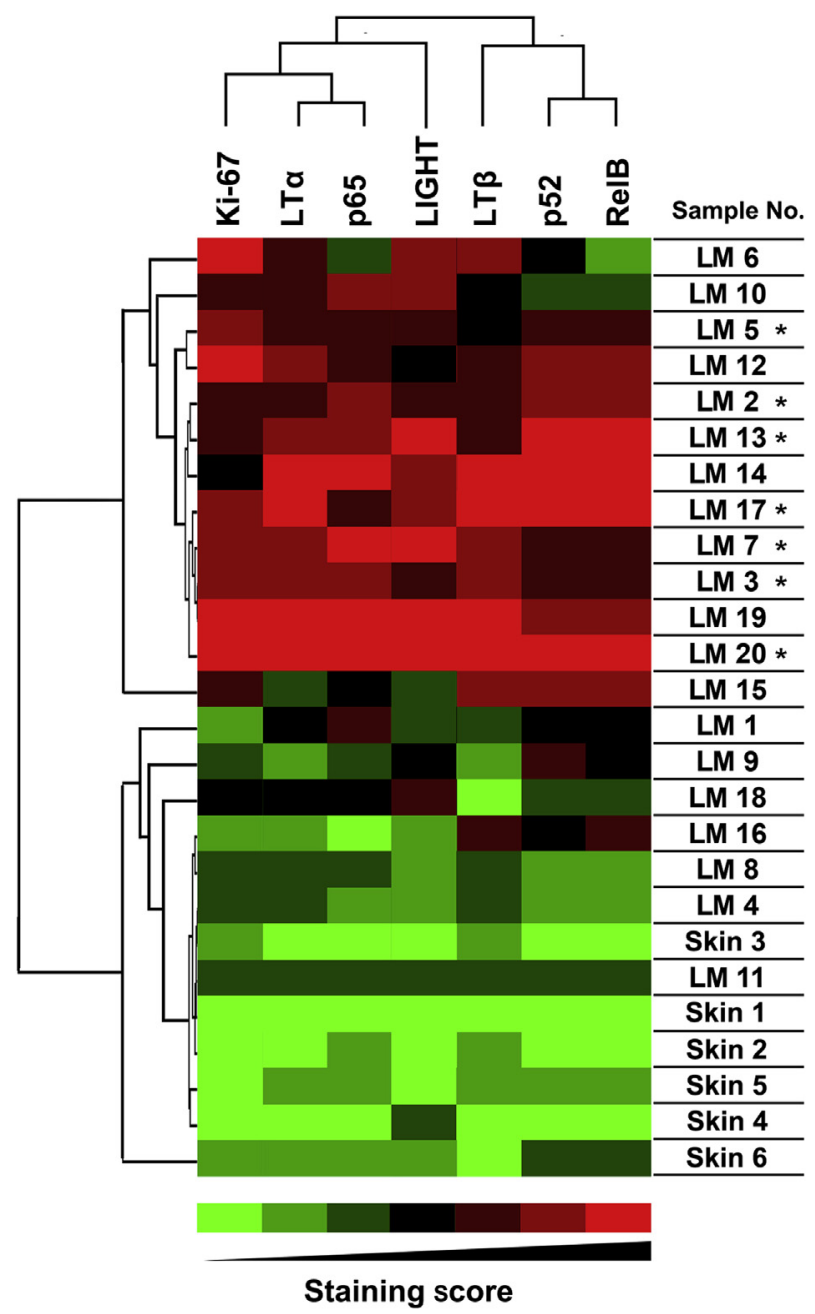

Figure 3 Hierarchical clustering analysis of human lymphatic malformation (LM) samples. Twenty LM samples (7 infected lesions and 13 uninfected lesions) and six normal skin tissue samples were analyzed. In the heat map, the relative relationship between these tested proteins (top) and tissue samples (left) is directly shown by the length and subdivision of the branches. The asterisks indicate LMs with infection. LIGHT, LT-related inducible ligand; LT, lymphotoxin.

infection affected the expression of LT receptors in human LECs directly. The fluorescence intensities of the LT receptors, including TNFR1, TNRF2, and LT $\beta R$, in HdLECs after 24-hour treatment with $1 \mu \mathrm{g} / \mathrm{mL}$ LPS were increased; the membrane expression of the receptors was dramatically enhanced (Figure 4B). The mRNA expression of TNFR1 and LT $\beta R$ was increased more than five times in HdLECs after LPS treatment by real-time PCR, and the mRNA expression of TNFR2 was also up-regulated significantly (Figure 4C). Moreover, the increased protein expression of LT receptors in LPS-treated HdLECs was verified by Western blot analysis (Figure 4, D and E).

To further investigate whether the increased expression of LT receptors led to higher responsivity to LTs in HdLECs, HdLECs were treated with recombinant LTs and LIGHT after pretreatment with LPS for 24 hours. The results from Western blot assays demonstrated that the expression of phosphorylated p65 increased in the LT $\alpha_{3}$-treated HdLECs, which was further enhanced by the pretreatment with LPS (Figure 4, F and G). Compared with the control group, the nuclear expression of RelB increased in $\mathrm{LT} \alpha_{1} \beta_{2^{-}}$and LIGHT-treated HdLECs, similar to pretreatment with LPS (Figure 4, H-K). These results proved that LPS enhanced the LT- and LIGHT-mediated activation of NF- $\kappa \mathrm{B}$ pathways in LECs because of the increased expression of LT receptors. These data also suggested that in infected LMs, LECs were more easily activated by LTs and LIGHT.

Given the close relationship between the expression of LTs and LIGHT and the proliferation of LECs in LMs by cluster analysis, we investigated whether LTs and LIGHT may promote the proliferation of LECs in vitro. In virtue of 5-ethyl-2'-deoxyuridine (EdU) incorporation assays, the effects of LTs and LIGHT on cell proliferation were examined. After treatment of $\mathrm{LT} \alpha_{3}$ and $\mathrm{LT} \alpha_{1} \beta_{2}$ for 24 hours, the ratio of HdLECs with positive EdU staining increased significantly from $24.93 \% \pm 1.03 \%$ to $43.93 \% \pm 1.10 \%$ and to $45.40 \% \pm 1.44 \%$, respectively. LIGHT also enhanced the proliferation of HdLECs (Figure 5, A and B). In addition, our data revealed that LPS significantly promoted the proliferation of HdLECs as the EdU-positive staining HdLECs increased from $24.93 \% \pm 1.03 \%$ to $42.08 \% \pm 0.64 \%$. To explore whether LT signaling pathways contributed to the LPS-induced proliferation of HdLECs, the expression of LT receptors, including TNFR1, TNFR2, and LT $\beta$ R, was suppressed using siRNAs; then, the proliferation of HdLECs treated with LPS was measured. Knockdown of the receptors was confirmed at both mRNA and protein levels (Figure 5, $\mathrm{C}-\mathrm{E}$ ). The proliferation of the HdLECs after siRNA treatment was measured in the presence of LPS. The results showed that knockdown of TNFR1 and LT $\beta$ R significantly reduced the LPS-enhanced proliferation of HdLECs (Figure 5, F and G). However, suppression of TNFR2 did not weaken the enhanced proliferation abilities of HdLECs treated with LPS. Knockdown of all three receptors further alleviated the proliferation of HdLECs. These data suggested that TNFR1- and LT $\beta R$-mediated signaling pathways are mainly involved in LPS-induced proliferation of LECs. Taken together, our results evidenced that LT- and LIGHT-mediated signaling pathways enhanced the proliferation of LECs, which might promote the development of LMs. Moreover, the above results suggested that infection might contribute to the progression of LMs by activation of LT signaling pathways through up-regulating LT receptors in LECs.

\section{LPS Up-Regulates LT and LIGHT Expression, Activates NF- $\kappa B$ Pathways, and Promotes the Development of LMs in a Rat Model}

To further clarify the role of LTs and LIGHT in LM development, rat models of LMs were established by 
A

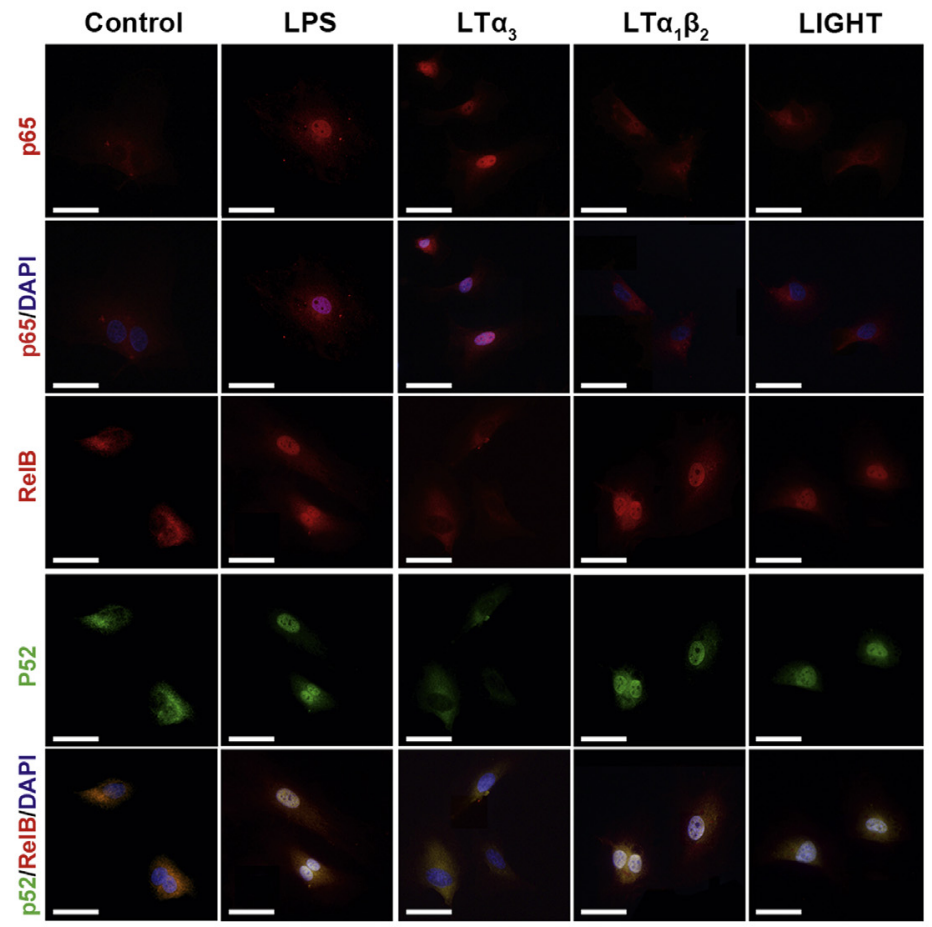

B

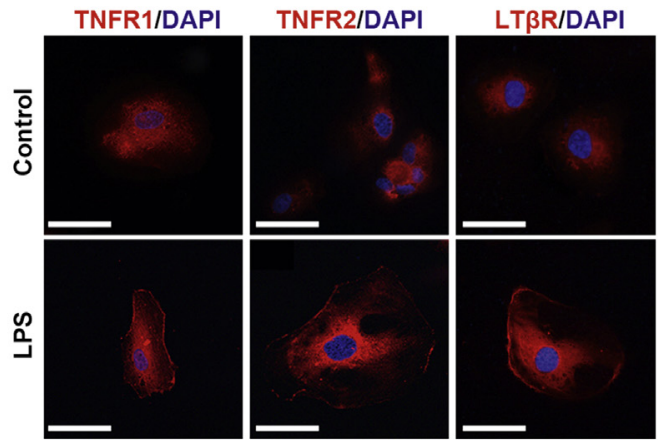

C

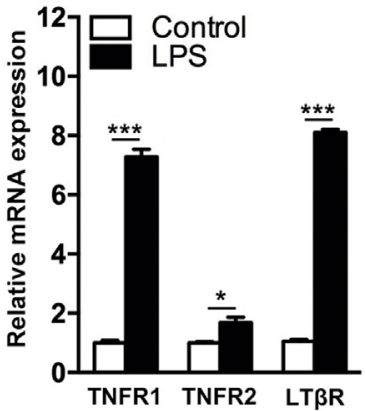

D

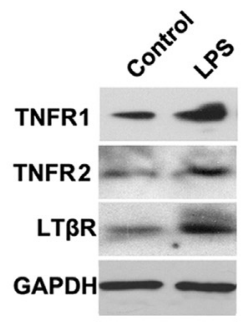

H

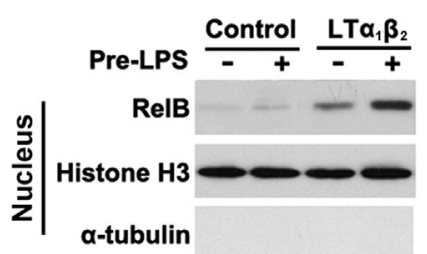

E

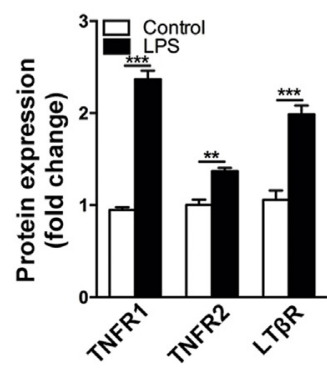

I

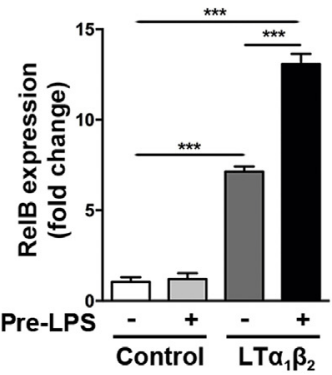

F

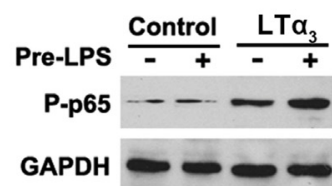

J

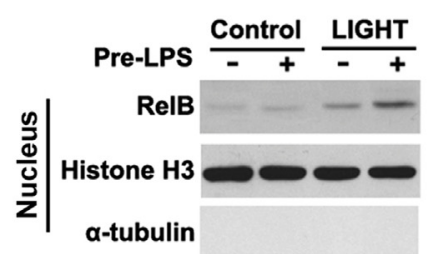

G

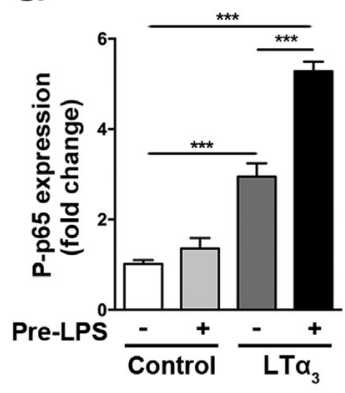

K

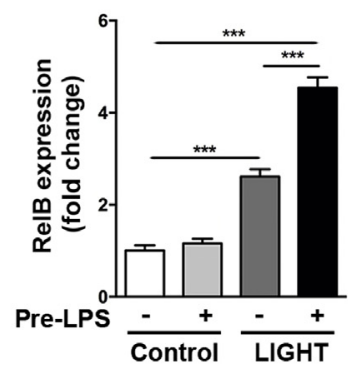

Figure 4 Activation of lymphotoxin (LT) signaling pathways in lipopolysaccharide (LPS)-treated human dermal lymphatic endothelial cells (HdLECs). A: Immunofluorescence of p65, p52, and RelB in HdLECs treated with LPS, recombinant LT $\alpha_{3}, L T \alpha_{1} \beta_{2}$, and LT-related inducible ligand (LIGHT) for 24 hours. B: Immunofluorescence of tumor necrosis factor receptor (TNFR) 1, TNFR2, and LT $\beta R$ in HdLECs treated with LPS for 24 hours. C: mRNA expression of LT receptors TNFR1, TNFR2, and LT $\beta R$ in LPS-treated HdLECs. D: Protein expression of TNFR1, TNFR2, and LT $\beta R$ in LPS-treated HdLECs. E: Quantitative analysis of LT receptor expression in LPS-treated HdLECs. F and G: HdLECs were pretreated with LPS for 24 hours, followed by treatment of recombinant LT $\alpha$ for another 24 hours. Then, the expression of phosphorylated p65 (p-p65) was examined by Western blot analyses (F) and quantified (G). H-K: After pretreatment of LPS for 24 hours, HdLECs were treated with recombinant $\mathrm{LT} \alpha_{1} \beta_{2}$ or LIGHT for another 24 hours. The nuclear proteins were harvested, and the expression of RelB was determined by Western blot analyses ( $\mathbf{H}$ and $\mathbf{J}$ ) and quantified (I and $\mathbf{K})$. Glyceraldehyde-3-phosphate dehydrogenase (GAPDH), histone H3, and $\alpha$-tubulin were used as loading control. Data are expressed as means $\pm \operatorname{SEM}(\mathbf{C}, \mathbf{E}, \mathbf{G}, \mathbf{I}$, and $\mathbf{K}) . n=3(\mathbf{C}-\mathbf{K}) .{ }^{*} P<0.05,{ }^{* *} P<0.01$, and ${ }^{* * *} P<0.001$. Scale bars: $100 \mu \mathrm{m}(\mathbf{A}) ; 50 \mu \mathrm{m}(\mathbf{B})$. 

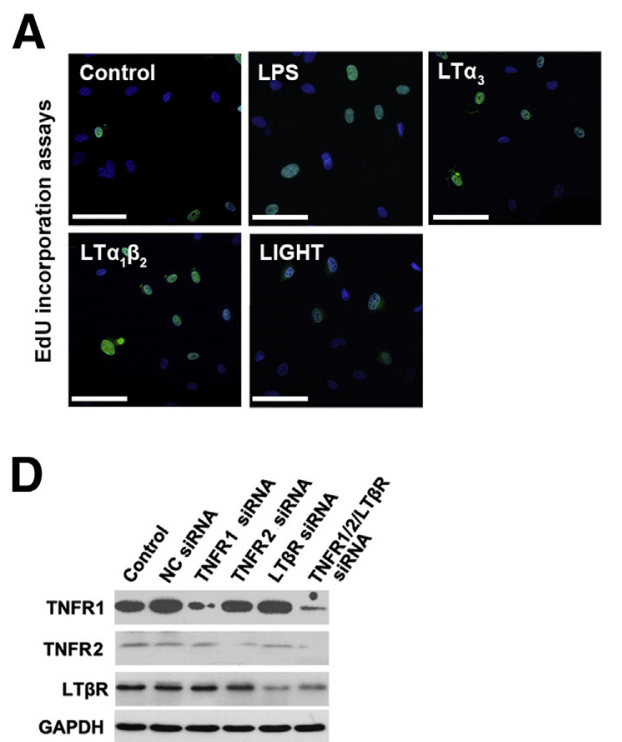

$\mathbf{F}$

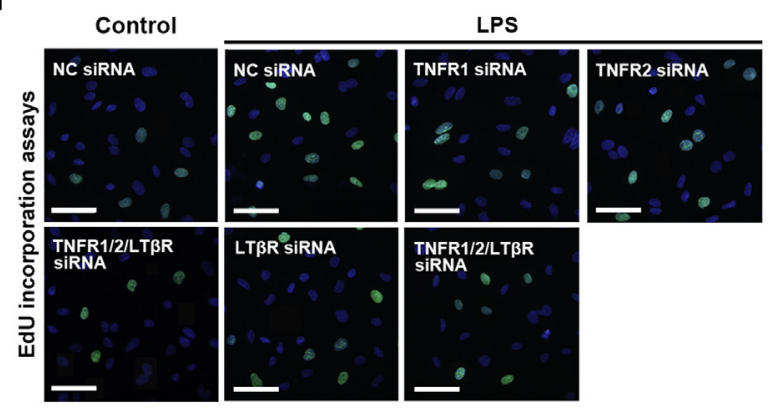

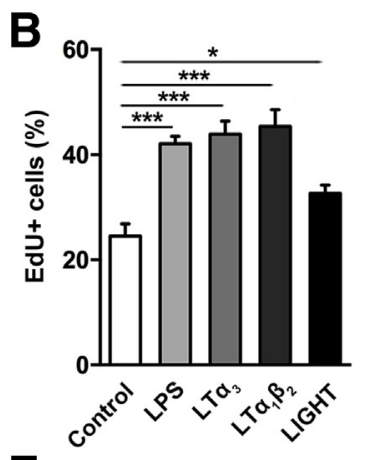

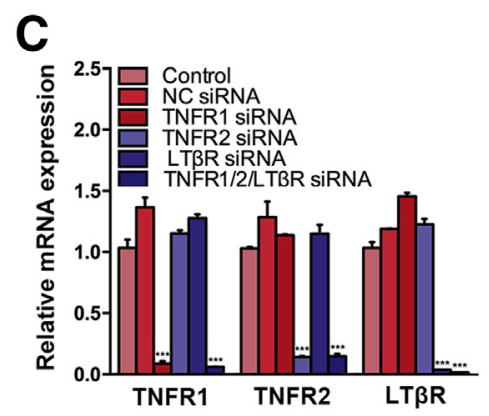

E

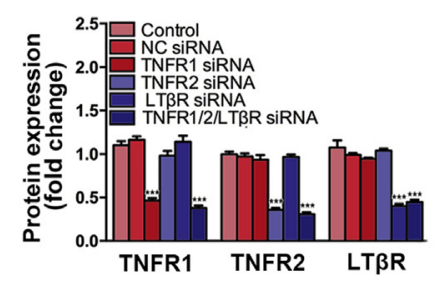

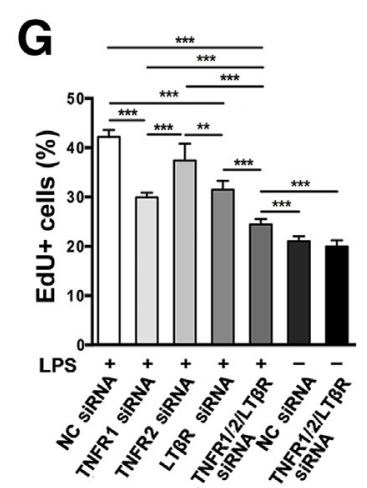

Figure 5 Lymphotoxin (LT) signaling pathways involved in lipopolysaccharide (LPS)-enhanced human dermal lymphatic endothelial cell (HdLEC) proliferation. A: 5-Ethyl-2'-deoxyuridine (EdU) incorporation assays of HdLECs treated with LPS, recombinant $L T \alpha_{3}$, $L T \alpha_{1} \beta_{2}$, and LT-related inducible ligand (LIGHT). B: Quantitative analysis of EdU incorporation assays. C and D: The mRNA (C) and protein (D) expression of tumor necrosis factor receptor (TNFR) 1 , TNFR2, and LT $\beta R$ in the HdLECs treated with TNFR1/2/LT $\beta R$ siRNAs. E: Quantitative analysis of LT receptor expression in the HdLECs treated with TNFR1/2/LT $\beta R$ siRNAs. F: Proliferation of the HdLECs was determined by EdU incorporation assays after treatment with LPS for 24 hours. G: Quantitative analysis of EdU incorporation assays. Data are expressed as means \pm SEM. $n=3(\mathbf{A}-\mathbf{G}) .{ }^{*} P<0.05,{ }^{*} * P<0.01$, and ${ }^{* * *} P<0.001$. Scale bars $=100 \mu \mathrm{m}(\mathbf{A}$ and $\mathbf{F})$. GAPDH, glyceraldehyde-3-phosphate dehydrogenase; NC, negative control.

injecting Freund's incomplete adjuvant into the neck region s.c., as previously reported. ${ }^{30}$ Considering the remarkable role of $\mathrm{LT} \alpha_{3}$ on HdLEC proliferation, Freund's incomplete adjuvant mixed with $120 \mathrm{ng} / \mathrm{mL}$ human recombinant $\mathrm{LT} \alpha_{3}$ was injected into the neck regions to determine the effects of LT $\alpha$ on the progression of LMs. Freund's incomplete adjuvant and $100 \mu \mathrm{g} / \mathrm{mL}$ LPS were injected to mimic the infected lesions, thus to explore whether LPS accelerated the progression of LMs via activation of LT signaling pathways. The rats only injected with Freund's incomplete adjuvant were set up as control. Treatments of recombinant $\mathrm{LT} \alpha_{3}$ or LPS led to bigger lesions compared with that of the control group, which was further supported by the quantitative measurement (Figure 6, A and B). Immunohistochemistry showed that the expression of LT $\alpha$ and LT $\beta$ was significantly up-regulated in LPS group lesions. The positive staining of LT $\alpha$ and LT $\beta$ can be found in the lining lymphatic endothelial cells of the enlarged channels and also in the stroma cells among the channels (Figure 6C). Moreover, the nuclear staining of p65 and p52 suggested the activation of classic and alternative NF- $\mathrm{BB}$ pathways in the LPS-treated lesions. In addition, strong staining of Ki-67 was also noticed in the serial sections, suggesting that the LTs and the following activated NF- $\kappa \mathrm{B}$ pathways might contribute to the proliferation of LECs in the models. Histoscores of the above biomarkers also supported the activation of LT signaling pathways (Figure 6D). However, notably, there was no statistical significance of p52 expression between the LPS group and the control group, suggesting that the classic NF- $\kappa$ B pathway might play a more important role during the progression of the lesion. The treatment with additional $\mathrm{LT} \alpha_{3}$ induced the nuclear expression of p65 rather than p52 (Figure 6E), in accordance with results above in which $\mathrm{LT} \alpha_{3}$ induced biological effects through the classic NF- $\mathrm{BB}$ 
A
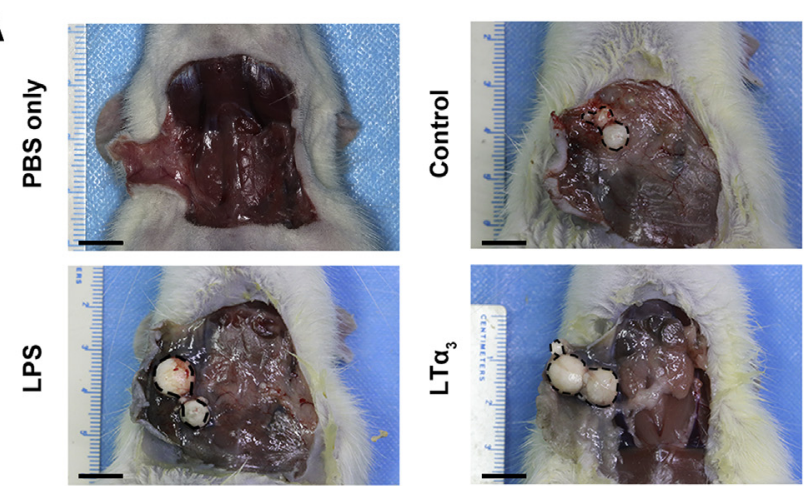

C
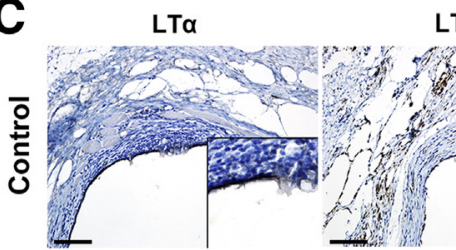

LT $\beta$
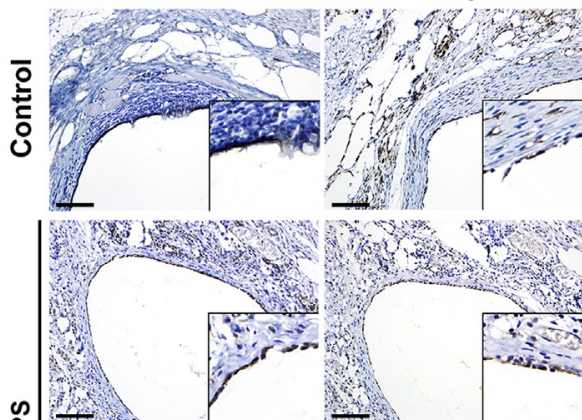

$\infty$
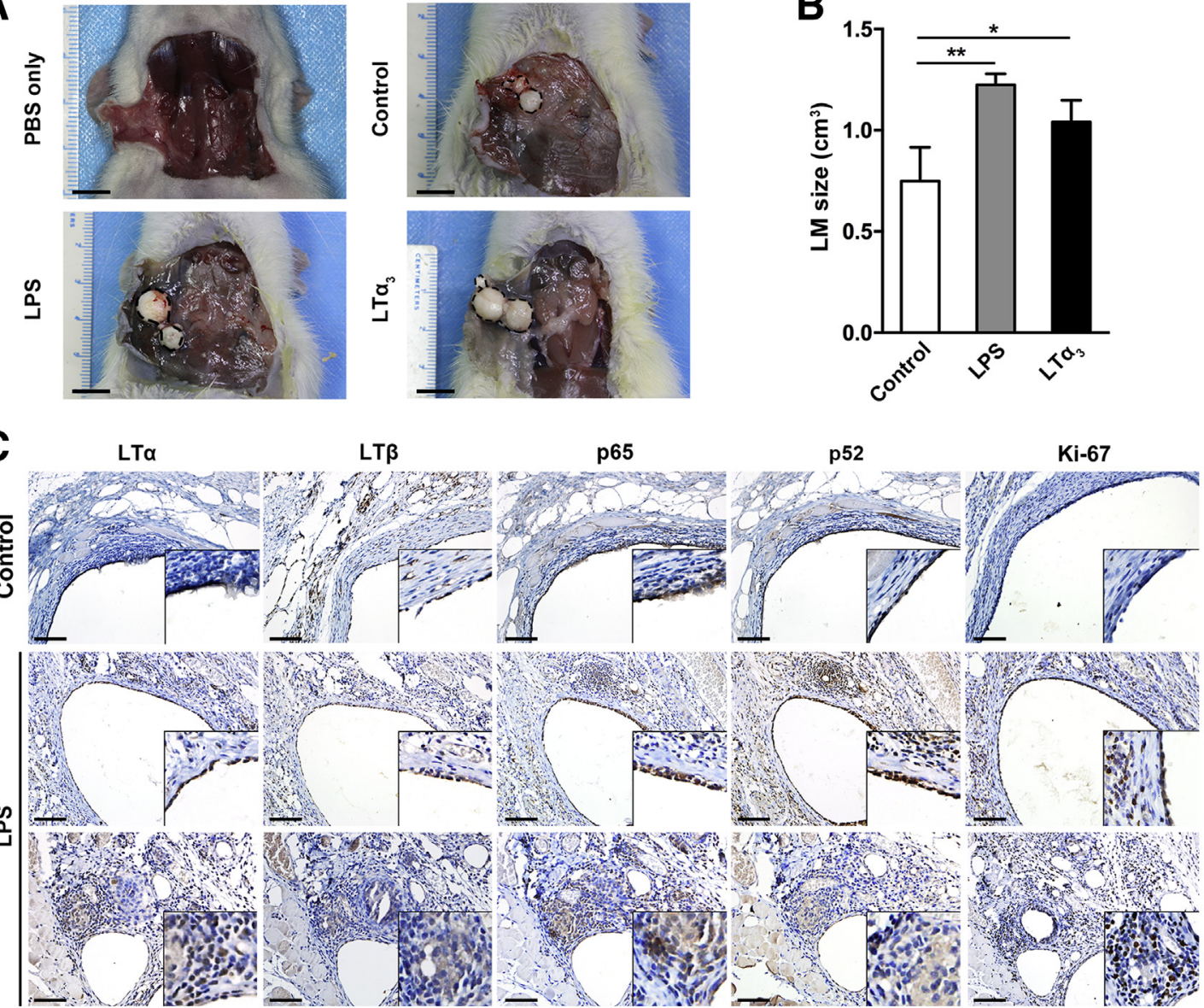

D

E
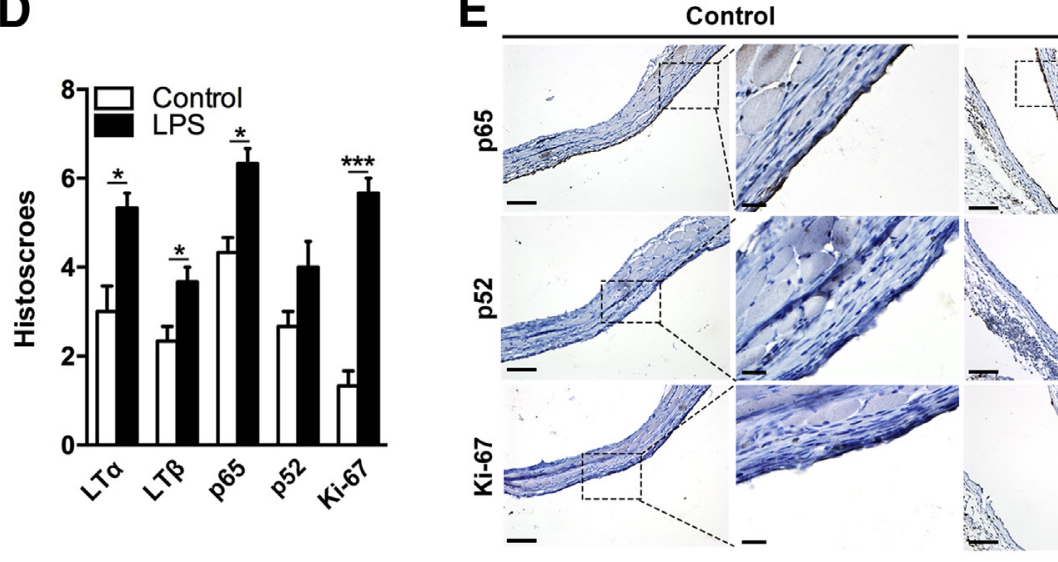

LTa Ab

$\mathbf{F}$
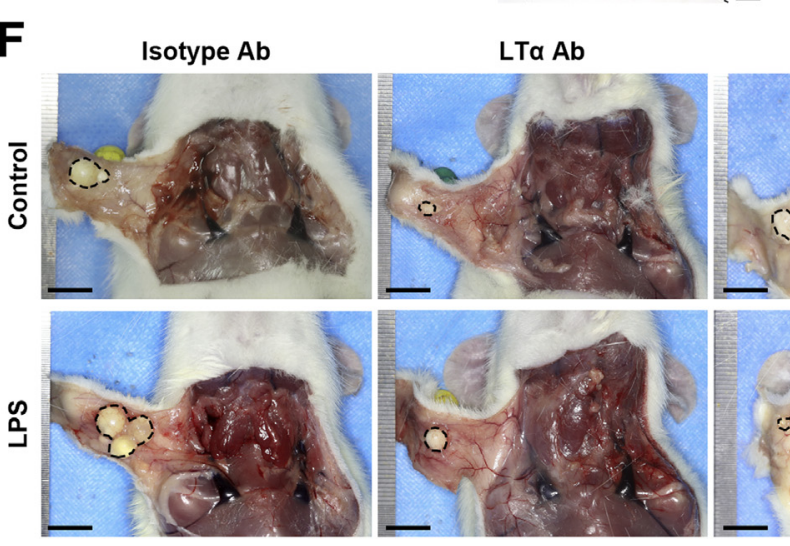

LT $\beta R$ Ab

G
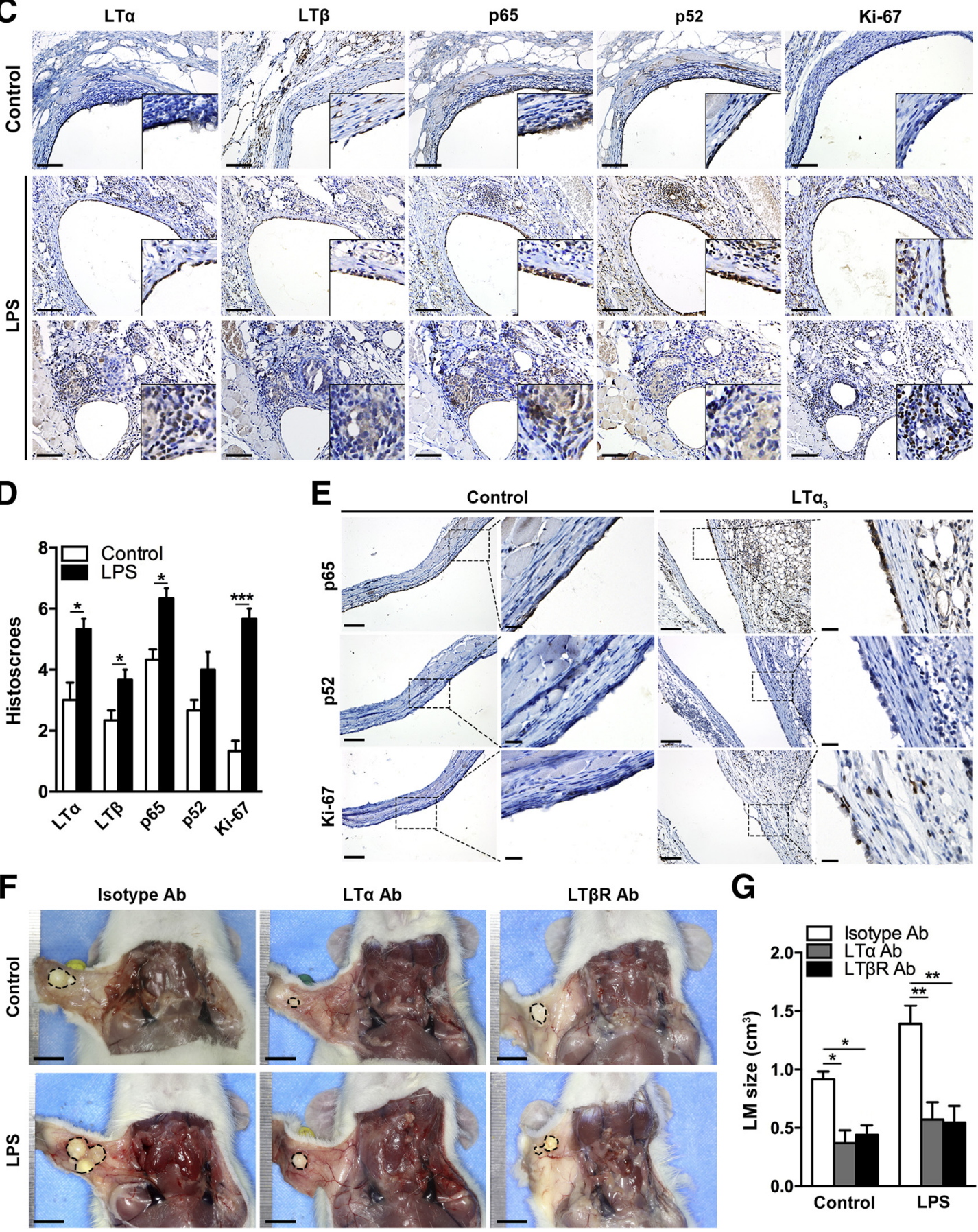
pathway. Treatment with additional $\mathrm{LT} \alpha_{3}$ also promoted the proliferation of the lining endothelial cells and stroma cells (Figure 6E). To investigate whether LPS-induced lesion progression was dependent on the LT signaling pathways, LT $\alpha$ - and LT $\beta$ R-neutralized antibodies were used to suppress LT-mediated signaling pathways. LT $\alpha$ - or LT $\beta$ R-neutralized antibodies impaired the growth of the lesions with LPS treatment (Figure 6F). Quantitative analysis further supported the suppressive effects of LT $\alpha$ and LT $\beta$ R inhibition on the progression of LMs (Figure 6G). These findings supported our hypothesis that inflammation induced by infection or other situations might contribute to the activation of the NF$\kappa \mathrm{B}$ pathway and accelerate the proliferation of LECs via upregulation of LTs in LMs, thus promoting disease progression.

\section{Discussion}

LMs were characterized by their enlarged and irregularly shaped lymphatic vessels, ${ }^{2}$ which led to draining dysfunction, deterioration of the symptom, and a high recurrence rate after traditional therapeutics, including surgery and sclerotherapy. ${ }^{40}$ Previous studies indicated LMs were chronic inflammatory diseases as lymphoid aggregates can be commonly found in lesions. ${ }^{10,11}$ Most of the lymphoid aggregates were shown to be TLOs, which were generally embedded in the sites affected with chronic inflammation. ${ }^{11}$ Lymphangiogenesis, formation of new lymphatic vessels, was a crucial event during the formation of TLOs because these vessels provided routes recruiting lymphocytes and released local edema. ${ }^{12,41}$ LTs and LIGHT were vital inflammation mediators that controlled the formation of TLOs by regulating the expression of lymphoid chemokines and high endothelial venule-related genes. ${ }^{18,42}$ Recent studies also reported that LTs and LIGHT were crucial for the development of lymphatic vessels in TLOs, as deficiency of LT $\beta R$ or LT $\alpha$ reduced the number and size of lymphatic vessels in TLOs. ${ }^{14}$ Moreover, it was reported that LT $\alpha$ regulated lymphangiogenesis by activating the stromal cells to produce VEGF-C and/or VEGF-A, both of which were powerful mediators of new lymphatic vessel formation. $^{43,44}$ Besides, another TNF ligand family member, TNF- $\alpha$, was reported to activate endothelial cells directly and promote their proliferation and migration, which led to lymphangiogenesis via the VEGF-C/VEGF receptor 3 signaling pathway. ${ }^{37}$ Clinical findings and our previous study indicated that infection and the accompanying chronic inflammation might be the crucial reason for the rapid development of LMs. ${ }^{10,11}$ Thus, we hypothesized that LTs and LIGHT might play important roles during the development of human LMs, especially in these infected lesions.

The expression of LTs, LIGHT, and their receptors in human specimens of LMs was first explored. Our results revealed the increasing concentration of LT $\alpha, \mathrm{LT} \beta$, and LIGHT, as well as positive staining of their receptors TNFR1, TNFR2, and LT $\beta R$ in LMs. During lymphangiogenesis, the positive feedback loop between stromal cells and lymphoid tissue inducer cells initiated by LTs and LIGHT was found in these lymphoid aggregates, as previously reported. ${ }^{45}$ In addition, the high expression of LT receptors was observed in lymphatic vessels of the LMs. In contrast, only slight staining of TNFR1 was observed in lymphatic vessels of the normal skins. These results indicated the potential role of LT signaling pathways in the progression of the malformation. To our best knowledge, the mechanism controlling the expression of LT receptors in endothelial cells has not been studied yet. Considering the role of inflammation in LM development, we speculated that inflammation might affect the expression of the LTs, LIGHT, and their receptors in LMs. Of interest, in infected LMs, our results revealed high LT and LIGHT expression in TLOs and increased expression of LT receptors in LECs. Besides, LPS significantly up-regulated the expression of LT receptors in HdLECs in vitro. However, the detailed molecular mechanism should be deeply explored.

By binding to their receptors, LTs and LIGHT activated $\mathrm{NF}-\kappa \mathrm{B}$ pathways in target cells, including the classic and alternative pathways, depending on the translocation of the transcription factors. It was reported that in most cases, the classic NF- $\kappa$ B pathway was activated by TNFR1, Toll-like receptor, or T- and B-cell receptors, ${ }^{23}$ whereas the alternative NF- $\kappa$ B pathway was generally activated by the LT $\beta$ R pathway. ${ }^{23}$ Herein, the activation of both classic and alternative NF- $\kappa B$ pathways was observed in the LECs of LM tissues, as proved by the nuclear translocation of $\mathrm{p} 65$ and $\mathrm{p} 52 / \mathrm{RelB}$, respectively. Moreover, results from in vitro assays revealed the nuclear translocation of p65 after recombinant $\mathrm{LT} \alpha_{3}$ treatment, and the nuclear translocation of p52/RelB after $\mathrm{LT} \alpha_{1} \beta_{2}$ and LIGHT stimulations, in line with the immunohistochemistry results in human LMs. NF- $\mathrm{BB}$ pathways played important roles in numerous physiological processes, including lymphangiogenesis. ${ }^{32,46}$ As reported, activation of NF- $\mathrm{KB}$ pathways in the tumor microenvironment may increase IL-8 expression and, thus, promote novel lymphatic vessel formation. ${ }^{47}$

Figure 6 Lipopolysaccharide (LPS) promotes the development of lymphatic malformations (LMs) through lymphotoxin (LT) signaling pathways in rat models. A: Representative images of LMs in rat models. Dashed lines illustrate LM lesions. B: Quantification of lesion size in different groups of rats. C: Immunohistochemical staining of LT $\alpha, L T \beta, p 65, p 52$, and Ki-67 in the lesions of different groups. Insets: Magnifications of local details. D: Histoscores of $\mathrm{LT} \alpha, \mathrm{LT} \beta, \mathrm{p} 65, \mathrm{p} 52$, and Ki-67 expression in the lesions of different groups. E: Immunohistochemical staining of p65, p52, and Ki-67 in the lesions treated with or without recombinant $L T \alpha_{3}$. F: Representative images of the lesions treated with neutralized $L T \alpha$ and $L T \beta R$ antibodies. Dashed lines illustrate $L M$ lesions. G: Quantification of lesion size in rats with indicated treatment. Data are expressed as means \pm SEM (B, D, and $\mathbf{G}) . n=5(\mathbf{A}-\mathbf{D}, \mathbf{F}$, and $\mathbf{G})$. ${ }^{*} P<0.05$, ${ }^{* *} P<0.01$, and ${ }^{* *} P<0.001$. Scale bars: $1 \mathrm{~cm}(\mathbf{A}$ and $\mathbf{F}) ; 50 \mu \mathrm{m}$ (C and $\left.\mathbf{E}\right) ; 8 \mu \mathrm{m}$ (E, insets). Ab, antibody; PBS, phosphate-buffered saline. 
Moreover, knockout of p50, an essential component for both classic and alternative NF- $\mathrm{KB}$ cascades, resulted in significantly reduced lymphatic vessel density in liver and lungs in mice. ${ }^{48}$ These studies suggest a close relationship between the NF- $\kappa \mathrm{B}$ and VEGF-C/VEGF receptor 3 pathways and activation of NF- $\kappa \mathrm{B}$ pathways to promote proliferation and migration of endothelial cells. Our results uncovered a significant correlation between p52 and Ki-67, as well as between p65 and Ki-67, suggesting that NF- $\mathrm{KB}$ pathway activation might enhance the proliferation of LECs and, hence, lymphangiogenesis. These findings were more evident in cluster analysis. In vitro EdU incorporation assays evidenced that recombinant $\mathrm{LT} \alpha_{3}, \mathrm{LT} \alpha_{1} \beta_{2}$, LIGHT, and LPS promoted LEC proliferation. More important, TNFR1- and LT $\beta$ R-mediated, but not TNFR2mediated, signaling pathways were found to be extremely important for LPS-induced LEC proliferation. This finding is consistent with previous studies that found that TNFR1, but not TNFR2, is more ubiquitously expressed in human tissues and serves as a major receptor for biological processes. ${ }^{49}$ These results indicated that LPS-induced LEC proliferation was at least partially dependent on the activation of LT signaling pathways. Moreover, the results from the cluster analysis showed that the infected lesions were clustered together with high expression of Ki-67, which was in accordance with our recent study, ${ }^{10}$ in which the close relationship between the inflammation in LMs and their progressions was demonstrated. Furthermore, using the LM rat model, we have shown that $\mathrm{LT} \alpha_{3}$ and LPS increase the volume of the lesion. We herein report that in LPS-treated lesions, the expression levels of LT $\alpha$ and LT $\beta$ are up-regulated significantly. Simultaneously, the activation of classic and alternative NF- $\kappa \mathrm{B}$ pathways was also evidenced in the samples. Additional treatment of recombinant $\mathrm{LT} \alpha_{3}$ induced up-regulation of proliferative marker Ki-67 in lymphatic endothelial cells, consistent with in vitro results. More important, suppression of LT signaling pathways using neutralizing antibodies, including LT $\alpha$ and LT $\beta$ R antibodies, attenuated LPS-stimulated progression of LMs. These results provided strong evidence supporting the fact that inflammation-related LT signaling pathways played crucial roles in the progression of LMs, which was possibly mediated by the classic and alternative NF- $\kappa$ B pathways. The close correlation between infection status and the location of human LMs might result from the high chance of infection in the oral cavity, which might be the main reason for refractoriness of the microcystic malformations.

In summary, we, for the first time, revealed the activation of LT signaling pathways in human LMs, which might contribute to the progression of LMs by promoting the proliferation of LECs. In addition, the results from in vitro and in vivo experiments suggested the close correlation between inflammation and activation of LT signaling pathways. This study sheds light on the role of LT signaling pathways in the pathogenesis of LMs, and it suggested that targeting LTs and LIGHT might be a potential treatment for LMs, especially for the infected malformations. However, the detailed mechanism by which LTs and LIGHT promote the lymphangiogenesis in LMs still needs to be explored.

\section{Acknowledgments}

J.-G.Y. performed immunohistochemical staining and cell experiments and wrote the manuscript; Y.-F.S. collected clinical data and performed animal experiments; Y.-F.Z. designed the study, analyzed data, revised the manuscript, and is the guarantor of the work; W.Z. designed the study, performed animal experiments, analyzed data, and revised the manuscript; K.-F.H. and J.-G.R. reviewed pathology samples; Z.-J.L. performed immunohistochemical staining; B.L. collected clinical data; all authors read and approved the final version submitted.

\section{Supplemental Data}

Supplemental material for this article can be found at http://dx.doi.org/10.1016/j.ajpath.2017.07.019.

\section{References}

1. Burrows PE, Mitri RK, Alomari A, Padua HM, Lord DJ, Sylvia MB, Fishman SJ, Mulliken JB: Percutaneous sclerotherapy of lymphatic malformations with doxycycline. Lymphat Res Biol 2008, 6:209-216

2. Boon LM, Vikkula M: Vascular anomalies. Edited by Wolff $\mathrm{K}$, Goldsmith LA, Katz LI, Gilchrest BA, Paller AS, Leffell DJ. In Fitzpatrick's Dermatology in General Medicine. ed 7. New York: McGraw-Hill Professional Publishing, 2008. pp. 1651-1666

3. Shiels WE 2nd, Kenney BD, Caniano DA, Besner GE: Definitive percutaneous treatment of lymphatic malformations of the trunk and extremities. J Pediatr Surg 2008, 43:136-139; discussion 140

4. Yadav P, De Castro DK, Waner M, Meyer L, Fay A: Vascular anomalies of the head and neck: a review of genetics. Semin Ophthalmol 2013, 28:257-266

5. Fageeh N, Manoukian J, Tewfik T, Schloss M, Williams HB Gaskin D: Management of head and neck lymphatic malformations in children. J Otolaryngol 1997, 26:253-258

6. Hancock BJ, St-Vil D, Luks FI, Di Lorenzo M, Blanchard H: Complications of lymphangiomas in children. J Pediatr Surg 1992, 27: 220-224. discussion 224-226

7. Jackson IT, Carreno R, Potparic Z, Hussain K: Hemangiomas, vascular malformations, and lymphovenous malformations: classification and methods of treatment. Plast Reconstr Surg 1993, 91:1216-1230

8. Bai Y, Jia J, Huang XX, Alsharif MJ, Zhao JH, Zhao YF: Sclerotherapy of microcystic lymphatic malformations in oral and facial regions. J Oral Maxillofac Surg 2009, 67:251-256

9. Edwards PD, Rahbar R, Ferraro NF, Burrows PE, Mulliken JB: Lymphatic malformation of the lingual base and oral floor. Plast Reconstr Surg 2005, 115:1906-1915

10. Zhang W, He KF, Yang JG, Ren JG, Sun YF, Zhao JH, Zhao YF Infiltration of M2-polarized macrophages in infected lymphatic malformations: possible role in disease progression. Br J Dermatol 2016, 175:102-112

11. Kirsh AL, Cushing SL, Chen EY, Schwartz SM, Perkins JA: Tertiary lymphoid organs in lymphatic malformations. Lymphat Res Biol 2011, 9:85-92 
12. Ruddle NH: Lymphatic vessels and tertiary lymphoid organs. J Clin Invest 2014, 124:953-959

13. Drayton DL, Liao S, Mounzer RH, Ruddle NH: Lymphoid organ development: from ontogeny to neogenesis. Nat Immunol 2006, 7: 344-353

14. Furtado GC, Marinkovic T, Martin AP, Garin A, Hoch B, Hubner W, Chen BK, Genden E, Skobe M, Lira SA: Lymphotoxin beta receptor signaling is required for inflammatory lymphangiogenesis in the thyroid. Proc Natl Acad Sci U S A 2007, 104:5026-5031

15. Schneider K, Potter KG, Ware CF: Lymphotoxin and LIGHT signaling pathways and target genes. Immunol Rev 2004, 202:49-66

16. Ware CF: Network communications: lymphotoxins, LIGHT, and TNF. Annu Rev Immunol 2005, 23:787-819

17. Ruddle NH: High endothelial venules and lymphatic vessels in tertiary lymphoid organs: characteristics, functions, and regulation. Front Immunol 2016, 7:491

18. Drayton DL, Ying X, Lee J, Lesslauer W, Ruddle NH: Ectopic LT $\alpha \beta$ directs lymphoid organ neogenesis with concomitant expression of peripheral node addressin and a HEV-restricted sulfotransferase. J Exp Med 2003, 197:1153-1163

19. Gräbner R, Lötzer K, Döpping S, Hildner M, Radke D, Beer M, Spanbroek R, Lippert B, Reardon CA, Getz GS: Lymphotoxin $\beta$ receptor signaling promotes tertiary lymphoid organogenesis in the aorta adventitia of aged ApoE-/- mice. J Exp Med 2009, 206:233-248

20. Mounzer RH, Svendsen OS, Baluk P, Bergman CM, Padera TP, Wiig H, Jain RK, McDonald DM, Ruddle NH: Lymphotoxin-alpha contributes to lymphangiogenesis. Blood 2010, 116:2173-2182

21. Zindl CL, Kim TH, Zeng M, Archambault AS, Grayson MH, Choi K, Schreiber RD, Chaplin DD: The lymphotoxin LT $\alpha 1 \beta 2$ controls postnatal and adult spleen marginal sinus vascular structure and function. Immunity 2009, 30:408-420

22. Madge LA, Kluger MS, Orange JS, May MJ: Lymphotoxin-alpha 1 beta 2 and LIGHT induce classical and noncanonical NF-kappa Bdependent proinflammatory gene expression in vascular endothelial cells. J Immunol 2008, 180:3467-3477

23. Hayden MS, Ghosh S: Signaling to NF-kappaB. Genes Dev 2004, 18: 2195-2224

24. Karin M, Greten FR: NF- $\kappa B$ : linking inflammation and immunity to cancer development and progression. Nat Rev Immunol 2005, 5:749-759

25. Xiao G, Rabson AB, Young W, Qing G, Qu Z: Alternative pathways of NF-KB activation: a double-edged sword in health and disease. Cytokine Growth Factor Rev 2006, 17:281-293

26. Ren JG, Chen G, Zhu JY, Zhang W, Sun YF, Jia J, Zhang J, Zhao YF: Downregulation of the transforming growth factor-beta/connective tissue growth factor 2 signalling pathway in venous malformations: its target potential for sclerotherapy. Br J Dermatol 2014, 171:242-251

27. Wang FQ, Chen G, Zhu JY, Zhang W, Ren JG, Liu H, Sun ZJ, Jia J, Zhao YF: M2-polarised macrophages in infantile haemangiomas: correlation with promoted angiogenesis. J Clin Pathol 2013, 66: $1058-1064$

28. Zhang W, Chen G, Wang FQ, Ren JG, Zhu JY, Cai Y, Zhao JH, Jia J, Zhao YF: Macrophages contribute to the progression of infantile hemangioma by regulating the proliferation and differentiation of hemangioma stem cells. J Invest Dermatol 2015, 135:3163-3172

29. Chen G, Ren JG, Zhang W, Sun YF, Wang FQ, Li RF, Zhang J, Zhao YF: Disorganized vascular structures in sporadic venous malformations: a possible correlation with balancing effect between Tie2 and TGF-beta. Sci Rep 2014, 4:5457

30. Sun Y, Jia J, Zhang W, Liu B, Zhang Z, Zhao Y: A reproducible invivo model of lymphatic malformation in rats. J Comp Pathol 2011, 145:390-398

31. Wigle JT, Harvey N, Detmar M, Lagutina I, Grosveld G, Gunn MD, Jackson DG, Oliver G: An essential role for PROX1 in the induction of the lymphatic endothelial cell phenotype. EMBO J 2002, 21:1505-1513
32. Flister MJ, Wilber A, Hall KL, Iwata C, Miyazono K, Nisato RE, Pepper MS, Zawieja DC, Ran S: Inflammation induces lymphangiogenesis through up-regulation of VEGFR-3 mediated by NF-kappaB and PROX1. Blood 2010, 115:418-429

33. Wu JK, Kitajewski C, Reiley M, Keung CH, Monteagudo J, Andrews JP, Liou P, Thirumoorthi A, Wong A, Kandel JJ, Shawber CJ: Aberrant lymphatic endothelial progenitors in lymphatic malformation development. PLoS One 2015, 10:e0117352

34. Brice G, Child AH, Evans A, Bell R, Mansour S, Burnand K, Sarfarazi M, Jeffery S, Mortimer P: Milroy disease and the VEGFR-3 mutation phenotype. J Med Genet 2005, 42:98-102

35. Suna S, Sakata Y, Shimizu M, Nakatani D, Usami M, Matsumoto S, Mizuno H, Ozaki K, Takashima S, Takeda H, Tanaka T, Hori M, Sato H: Lymphotoxin-alpha3 mediates monocyte-endothelial interaction by TNFR I/NF-kappaB signaling. Biochem Biophys Res Commun 2009, 379:374-378

36. Ganeff C, Remouchamps C, Boutaffala L, Benezech C, Galopin G, Vandepaer S, Bouillenne F, Ormenese S, Chariot A, Schneider P, Caamano J, Piette J, Dejardin E: Induction of the alternative NFkappaB pathway by lymphotoxin alphabeta (LTalphabeta) relies on internalization of LTbeta receptor. Mol Cell Biol 2011, 31: 4319-4334

37. Ji H, Cao R, Yang Y, Zhang Y, Iwamoto H, Lim S, Nakamura M, Andersson P, Wang J, Sun Y, Dissing S, He X, Yang X, Cao Y: TNFR1 mediates TNF-alpha-induced tumour lymphangiogenesis and metastasis by modulating VEGF-C-VEGFR3 signalling. Nat Commun 2014, 5:4944

38. Bhattacharyya S, Borthakur A, Dudeja PK, Tobacman JK: Lipopolysaccharide-induced activation of NF-kappaB non-canonical pathway requires BCL10 serine 138 and NIK phosphorylations. Exp Cell Res 2010, 316:3317-3327

39. Yang F, Tang E, Guan K, Wang C-Y: IKK $\beta$ plays an essential role in the phosphorylation of RelA/p65 on serine 536 induced by lipopolysaccharide. J Immunol 2003, 170:5630-5635

40. Hogeling M, Adams S, Law J, Wargon O: Lymphatic malformations: clinical course and management in 64 cases. Australas J Dermatol 2011, 52:186-190

41. Kataru RP, Jung K, Jang C, Yang H, Schwendener RA, Baik JE, Han SH, Alitalo K, Koh GY: Critical role of CD11b+ macrophages and VEGF in inflammatory lymphangiogenesis, antigen clearance, and inflammation resolution. Blood 2009, 113:5650-5659

42. Kratz A, Campos-Neto A, Hanson MS, Ruddle NH: Chronic inflammation caused by lymphotoxin is lymphoid neogenesis. J Exp Med 1996, 183:1461-1472

43. Karkkainen MJ, Haiko P, Sainio K, Partanen J, Taipale J, Petrova TV, Jeltsch M, Jackson DG, Talikka M, Rauvala H: Vascular endothelial growth factor $\mathrm{C}$ is required for sprouting of the first lymphatic vessels from embryonic veins. Nat Immunol 2004, 5:74-80

44. Halin C, Tobler NE, Vigl B, Brown LF, Detmar M: VEGF-A produced by chronically inflamed tissue induces lymphangiogenesis in draining lymph nodes. Blood 2007, 110:3158-3167

45. Gommerman JL, Browning JL: Lymphotoxin/light, lymphoid microenvironments and autoimmune disease. Nat Rev Immunol 2003, 3: 642-655

46. Moynagh PN: The NF-кB pathway. J Cell Sci 2005, 118:4589-4592

47. Mu H, Calderone TL, Davies MA, Prieto VG, Wang H, Mills GB, BarEli M, Gershenwald JE: Lysophosphatidic acid induces lymphangiogenesis and IL-8 production in vitro in human lymphatic endothelial cells. Am J Pathol 2012, 180:2170-2181

48. Flister MJ, Volk LD, Ran S: Characterization of PROX1 and VEGFR-3 expression and lymphatic phenotype in normal organs of mice lacking p50 subunit of NF-kappaB. Microcirculation 2011, 18:85-101

49. Croft M, Benedict CA, Ware CF: Clinical targeting of the TNF and TNFR superfamilies. Nat Rev Drug Discov 2013, 12:147-168 